\title{
Swift observations of GRB 060614: an anomalous burst with a well behaved afterglow ${ }^{\star}$
}

\author{
V. Mangano ${ }^{1}$, S. T. Holland ${ }^{2,3}$, D. Malesani ${ }^{4}$, E. Troja ${ }^{1,5,6}$, G. Chincarini ${ }^{7,8}$, B. Zhang ${ }^{9}$, V. La Parola ${ }^{1,7}$, P. J. Brown ${ }^{10}$, \\ D. N. Burrows ${ }^{10}$, S. Campana ${ }^{8}$, M. Capalbi ${ }^{11}$, G. Cusumano ${ }^{1}$, M. Della Valle ${ }^{12}$, N. Gehrels ${ }^{2}$, P. Giommi ${ }^{11}$, D. Grupe ${ }^{10}$, \\ C. Guidorzi ${ }^{7,8}$, T. Mineo ${ }^{1}$, A. Moretti ${ }^{8}$, J. P. Osborne ${ }^{5}$, S. B. Pandey ${ }^{13}$, M. Perri ${ }^{11}$, P. Romano ${ }^{7,8}$, \\ P. W. A. Roming ${ }^{10}$, and G. Tagliaferri ${ }^{8}$
}

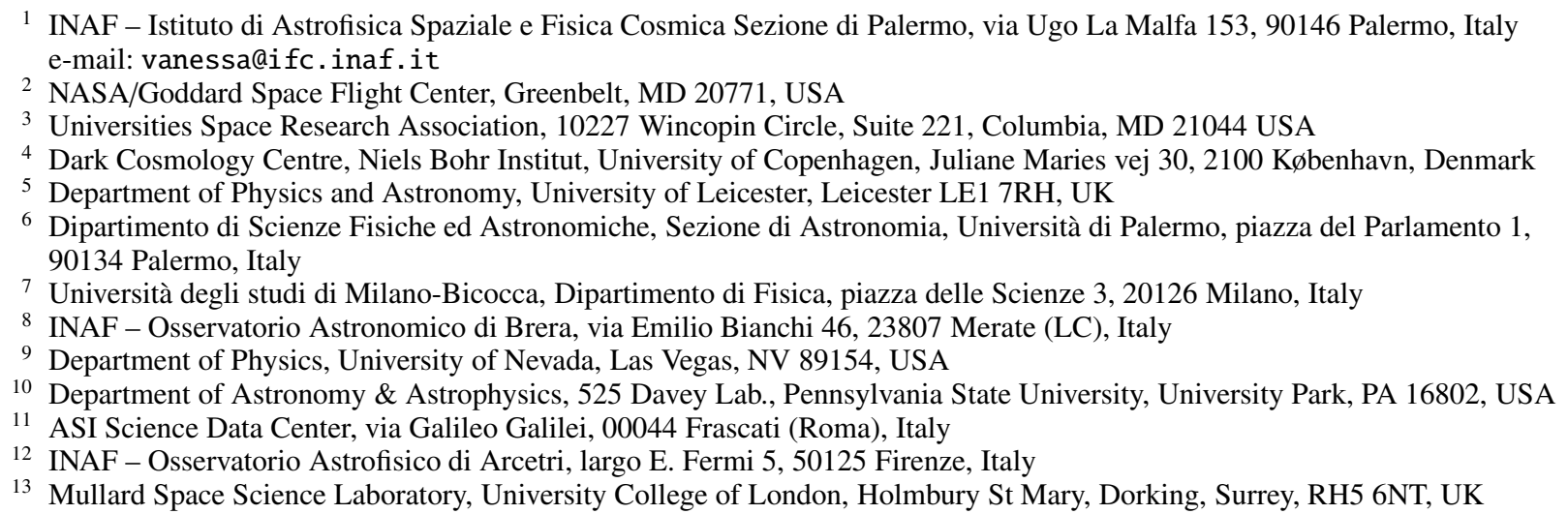

Received 2 February 2007 / Accepted 13 April 2007

\section{ABSTRACT}

\begin{abstract}
GRB 060614 is a remarkable gamma-ray burst (GRB) observed by Swift with puzzling properties, which challenge current progenitor models. In particular, the lack of any bright supernova (SN) down to very strict limits and the vanishing spectral lags during the whole burst are typical of short GRBs, strikingly at odds with the long (102 s) duration of this event. Here we present detailed spectral and temporal analysis of the Swift observations of GRB 060614. We show that the burst presents standard optical, ultraviolet and X-ray afterglows, detected beginning $4 \mathrm{ks}$ after the trigger. An achromatic break is observed simultaneously in the optical and X-ray bands, at a time consistent with the break in the $R$-band light curve measured by the VLT. The achromatic behaviour and the consistent postbreak decay slopes make GRB 060614 one of the best examples of a jet break for a Swift burst. The optical and ultraviolet afterglow light curves have also an earlier break at $29.7 \pm 4.4 \mathrm{ks}$, marginally consistent with a corresponding break at $36.6 \pm 2.4 \mathrm{ks}$ observed in the X-rays. In the optical, there is strong spectral evolution around this break, suggesting the passage of a break frequency through the optical/ultraviolet band. The very blue spectrum at early times suggests this may be the injection frequency, as also supported by the trend in the light curves: rising at low frequencies, and decaying at higher energies. The early X-ray light curve (from 97 to $480 \mathrm{~s}$ ) is well interpreted as the X-ray counterpart of the burst extended emission. Spectral analysis of the BAT and XRT data in the $\sim 80 \mathrm{~s}$ overlap time interval show that the peak energy of the burst has decreased to as low as $8 \mathrm{keV}$ at the beginning of the XRT observation. Spectral analysis of following XRT data shows that the peak energy of the burst continues to decrease through the XRT energy band and exits it at about $500 \mathrm{~s}$ after the trigger. The average peak energy $E_{\mathrm{p}}$ of the burst is likely below the BAT energy band $(<24 \mathrm{keV}$ at the $90 \%$ confidence level) but larger than $8 \mathrm{keV}$. The initial group of peaks observed by BAT $(\sim 5 \mathrm{~s})$ is however distinctly harder than the rest of the prompt emission, with a peak energy of about $300 \mathrm{keV}$ as measured by Konus Wind. Considering the time-averaged spectral properties, GRB 060614 is consistent with the $E_{\mathrm{iso}}-E_{\mathrm{p}}^{\text {rest }}, E_{\gamma}-E_{\mathrm{p}}^{\text {rest }}$, and $L_{\mathrm{p} \text {,iso }}-E_{\mathrm{p}}^{\text {rest }}$ correlations.
\end{abstract}

Key words. gamma rays: bursts - X-rays: individuals: GRB 060614

\section{Introduction}

The Swift Gamma-Ray Burst Explorer (Gehrels et al. 2004), successfully launched on 2004 Nov. 20, is a multi-wavelength space observatory with a payload that includes one wide-field instrument, the Burst Alert Telescope (BAT, 15-350 keV energy band; Barthelmy et al. 2005b), and two narrow-field instruments (NFIs), the X-Ray Telescope (XRT, 0.2-10 keV;

* Tables 1 and 2 are only available in electronic form at http: //www . aanda.org
Burrows et al. 2005) and the Ultraviolet/Optical Telescope (UVOT, 1700-6500 Å; Roming et al. 2005). BAT has been designed for burst detection and localization to $\$ 3^{\prime}$ accuracy. It triggers an autonomous slew of the observatory to point the two narrow-field instruments, typically within $100 \mathrm{~s}$ from the burst onset. XRT can provide $\lesssim 5^{\prime \prime}$ positions, while UVOT further refines the afterglow localization to $\sim 00^{\prime} .5$.

Swift-BAT triggered on GRB 060614 (trigger 214805) on 2006 June 14 at 12:43:48 UT, and located it at the coordinates $\mathrm{RA}_{\mathrm{J} 2000}=21^{\mathrm{h}} 23^{\mathrm{m}} 27^{\mathrm{s}}, \operatorname{Dec}_{\mathrm{J} 2000}=-53^{\circ} 02^{\prime} 02^{\prime \prime}$, with an 
uncertainty of 3' (90\% containment, including systematic uncertainty; Parsons et al. 2006). The spacecraft executed an automatic and immediate slew to the burst location and started observing with XRT and UVOT 91 and $101 \mathrm{~s}$ after the trigger, respectively. The afterglow emission was monitored for more than 30 days.

The BAT mask-weighted light curve showed a multi-peaked structure beginning with an initial $\sim 5 \mathrm{~s}$ series of hard, bright peaks followed by a fainter, softer and highly variable extended prompt emission. The observed fluence in the $15-150 \mathrm{keV}$ energy band was estimated at the level of $(2.17 \pm 0.04) \times$ $10^{-5} \mathrm{erg} \mathrm{cm} \mathrm{cm}^{-2}$ for a burst duration of $T_{90}=102 \pm 5 \mathrm{~s}$ (15-350 keV; Barthelmy et al. 2006). This is one of the highest fluences ever observed for a Swift bursts located by BAT.

XRT found a very bright $\left(\sim 1300\right.$ counts $\left.\mathrm{s}^{-1}\right)$ uncatalogued source inside the BAT error circle. The object showed an initial fast exponential decay and then a flattening at the level of $\sim 0.2$ counts $\mathrm{s}^{-1}$, followed by a steepening to a standard afterglow evolution. The initial decay was accompanied by strong hard-to-soft spectral evolution (Mangano et al. 2006).

The optical counterpart, originally detected in the White $(160-650 \mathrm{~nm})$ filter at the level of $18.4 \pm 0.5 \mathrm{mag}$, was later visible in all of the UVOT bands (White, $V, B, U, U V W 1, U V M 2$, and $U V W 2$ filters). The detection in the $U V W 2$ filter allows us to set a strong upper limit to the burst redshift $(z<1.3$ at the $99.99 \%$ confidence level; Gehrels et al. 2006) and implies very low dust extinction.

Konus-Wind was also triggered by GRB 060614 at 12:43:51.59 UT, $\sim 4 \mathrm{~s}$ after the BAT trigger. The spectrum of the first group of intense peaks was fitted in the $20 \mathrm{keV}-2 \mathrm{MeV}$ energy range by a power law with an exponential cut-off model $\left(\chi^{2} /\right.$ d.o.f. $\left.=73 / 59\right)$. The derived photon index was $1.57_{-0.14}^{+0.12}$ with a peak energy $E_{\mathrm{p}}=302_{-85}^{+214} \mathrm{keV}$. The spectrum of the remaining part of the prompt emission was described by a simple power law model with photon index $2.13 \pm 0.05$. The total fluence in the $20 \mathrm{keV}-2 \mathrm{MeV}$ energy band was $4.09_{-0.34}^{+0.18} \times$ $10^{-5} \mathrm{erg} \mathrm{cm}^{-2}$ (Golenetskii et al. 2006).

Ground-based optical and infrared follow-up observations were performed with several instruments. Optical/infrared imaging, made with the ANDICAM instrument on the $1.3 \mathrm{~m}$ telescope at the Cerro Tololo Inter-American Observatory (CTIO), revealed the GRB 060614 afterglow $\sim 15.5 \mathrm{~h}$ after the trigger both in the $I$ and $J$ bands (Cobb 2006; Cobb et al. 2006). In the $R$ band, the afterglow was first detected by the Siding Spring Observatory (SSO) $1 \mathrm{~m}$ telescope, brightening from a magnitude of $R=20.2 \pm 0.3 \sim 25 \mathrm{~min}$ after the BAT trigger to $R=18.8 \pm$ 0.1 after $\sim 6 \mathrm{~h}$ (Schmidt et al. 2006). Later observations were performed by the Watcher $0.4 \mathrm{~m}$ Telescope located in Boyden Observatory, South Africa $(R=19.0 \pm 0.3,7.1 \mathrm{~h}$ after the trigger; French et al. 2006), by the ESO VLT-UT1 $(R=19.3 \pm$ 0.2 after $\sim 14.4$ h; Malesani et al. 2006; Della Valle et al. 2006) and by the Danish $1.5 \mathrm{~m}$ Telescope equipped with the Danish Faint Object Spectrograph and Camera (DFOSC; Thoene et al. 2006; Fynbo et al. 2006b). The last two monitored the source for several weeks. The observed flattening of the optical and infrared emission few days after the burst was attributed to the host galaxy by Fynbo et al. (2006a) and Cobb \& Bailyn (2006).

Based on the detection of the host galaxy emission lines, a redshift of $z=0.125$ was proposed by Price et al. (2006) and confirmed by Fugazza et al. (2006). The GRB host is a faint $\left(M_{V}=-15.5\right)$ star-forming galaxy (Della Valle et al. 2006; Fynbo et al. 2006b) with a specific star formation rate at the low end of the distribution for long GRB hosts (Christensen et al. 2004; Sollerman et al. 2005). The GRB counterpart is located in the outskirts of the host (Gal-Yam et al. 2006). The probability of chance alignment between the GRB and this galaxy has been carefully estimated to be as low as $5.6 \times 10^{-6}$ by Gal-Yam et al. (2006).

The long monitoring campaigns of the ESO VLT and of the Danish $1.5 \mathrm{~m}$ Telescope at La Silla in Chile, and the target of opportunity observations of the Hubble Space Telescope (HST) did not detect any SN component emerging out of the host galaxy light (Della Valle et al. 2006; Fynbo et al. 2006b; Gal-Yam et al. 2006). Any associated SN had to be more than 100 times fainter than events previously known to be associated with long GRBs (SN1998bw/GRB980425, Galama et al. 1998; SN2003dh/GRB030329, Stanek et al. 2003; Hjorth et al. 2003; SN2003lw/GRB031203, Malesani et al. 2004; SN2006aj/GRB 060218, Campana et al. 2006a). These robust limits might suggest either that GRB 060614 has been produced during a merger process, or during the explosion of a "fall back" SN (Nomoto et al. 2004), or by a "dark hypernova" (Nomoto et al. 2007). Recently, Tominaga et al. (2007) showed through numerical simulations that jet-induced explosions in metal-poor massive stars can produce faint type-II SNe or dark HNe consistent with existing upper limits.

Gehrels et al. (2006) noted that the first 5 s of the prompt emission, including the brightest peaks of the BAT light curve, show many sub-pulses with time lags consistent with zero, like short bursts and unlike long GRBs, which usually have positive lags (Norris 2002). In particular, in the peak luminosity-time lag plane, the brightest peak of GRB 060614 lies in the region occupied by short bursts (Gehrels et al. 2006). Another similarity with short bursts is the structure of the BAT light curve, starting with a series of bright, hard peaks, followed by a group of lower luminosity, softer peaks and a smooth tail. This is reminiscent of the long soft hump seen in several short GRBs such as GRB 050709 (Villasenor et al. 2005), GRB 050724 (Barthelmy et al. 2005c; Campana et al. 2006b), GRB 051227 (Barthelmy et al. 2005a), GRB 060121 (HETE-2; Donaghy et al. 2006), GRB 061006 (Krimm et al. 2006), GRB 061210 (Palmer et al. 2006), and a few bursts in the BATSE sample (Norris \& Bonnell 2006; see also Lazzati et al. 2001). Indeed Zhang et al. (2007) showed that GRB 060614, were it 8 times less energetics, would have been detected by BATSE as a marginal short-duration GRB and by Swift as an analog to GRB 050724.

However, current models of compact binary merger progenitors can hardly account for $\sim 100 \mathrm{~s}$ prolonged emission (Rosswog et al. 2003; Lee et al. 2004), even if the complexity of the physics involved does not allow to set firm conclusions yet. In addition we note that recently Amati et al. (2007) have shown that GRB 060614 is consistent with the $E_{\mathrm{p}}$ vs. $E_{\text {iso }}$ relationship which applies only to long-duration GRBs (Amati 2006). For these reasons GRB 060614 has been proposed to belong to a new class of GRBs sharing observational properties with both the long and short GRBs (Gehrels et al. 2006) and possibly coming from different progenitors (King et al. 2007).

Here we present a detailed analysis of Swift observations of GRB 060614. Details on the BAT, XRT and UVOT observations are given in Sect. 2; data reduction is described in Sects. 2.1, 2.2, and 2.3; the temporal and spectral analysis results are reported in Sect. 3, a summary of the results and discussion are presented in Sect. 4. Conclusions are drawn in Sect. 5.

Throughout this paper the quoted uncertainties are given at $90 \%$ confidence level for one interesting parameter (i.e., $\Delta \chi^{2}=$ 2.71) unless otherwise stated. Times are referred to the BAT 


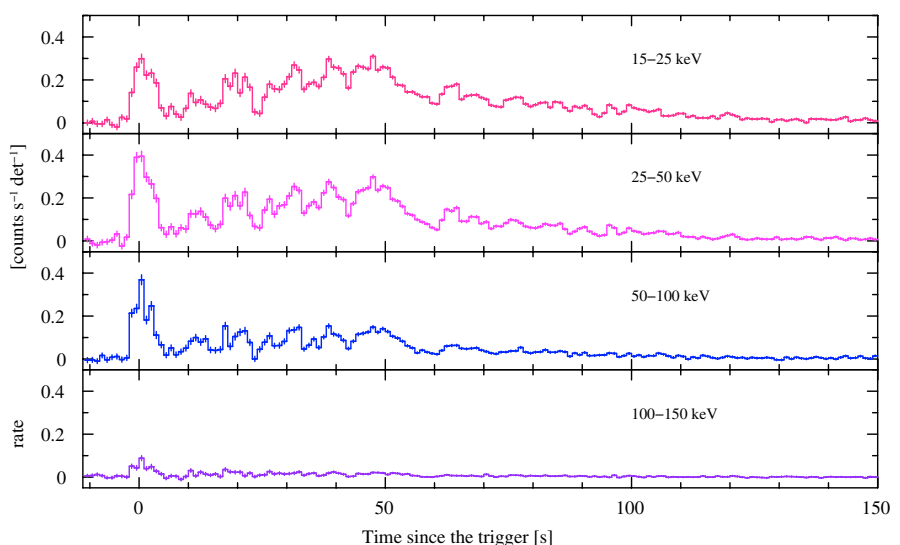

Fig. 1. BAT light curves in four energy bands.

trigger $T_{0}, t=T-T_{0}$, unless otherwise specified. We also adopt the notation $F_{v}(v, t) \propto t^{-\alpha} v^{-\beta}$ for the afterglow monochromatic flux as a function of time, where $v$ represents the frequency of the observed radiation. The energy index $\beta$ is related to the photon index $\Gamma$ according to $\beta=\Gamma-1$. We adopt a standard cosmology model with $H_{0}=70 \mathrm{~km} \mathrm{~s}^{-1} \mathrm{Mpc}^{-1}, \Omega_{\mathrm{M}}=0.3, \Omega_{\Lambda}=0.7$.

\section{Observations and data reduction}

The list of all BAT, XRT and UVOT observations of GRB 060614 used for the present analysis is shown in Tables 1 and 2. XRT and UVOT follow-up lasted 51 days and consisted of 38 sequences numbered from 0 to 37 . The total XRT exposure spent on GRB 060614 was 514 ks. For both XRT and UVOT, merging data of many sequences was necessary to attain source detection at later times. In these cases only information about the merged data is reported.

\subsection{BAT}

The BAT event data were re-analyzed using the standard BAT analysis software (Swift2.4) as described in the Swift BAT Ground Analysis Software Manual (Krimm et al. 2004). This incorporates post-launch updates to the BAT response and to the effective area and includes the systematic error vector to be applied to the spectrum. The ground analysis of BAT data gave a refined position of the burst at $\mathrm{RA}_{\mathrm{J} 2000}=21^{\mathrm{h}} 23^{\mathrm{m}} 31^{\mathrm{s}} .8$, $\operatorname{Dec}_{\mathrm{J} 2000}=-53^{\circ} 02^{\prime} 04^{\prime \prime}$. 4 , with an error radius of $1^{\prime}(90 \%$ containment, including systematics; Barthelmy et al. 2006). Maskweighted BAT light curves were created in the standard 4 energy bands $(15-25,25-50,50-100,100-150 \mathrm{keV})$, and in the total $15-150 \mathrm{keV}$ band (Figs. 1 and 2) at $1 \mathrm{~s}$ time resolution. These light curves show an unusual multi-peaked burst structure that begins with an initial bright group of peaks with a $5 \mathrm{~s}$ FWHM duration, followed by a set of fainter and somewhat softer peaks that increase in intensity. At about $T_{0}+60 \mathrm{~s}$, the light curve shows a hint of a last faint peak and then decays smoothly. Sub-pulses on the timescale of tens of $\mathrm{ms}$ are present, especially during the initial 5-8 s (see the inset in Fig. 2). The duration of the burst can be estimated as $T_{90}=102 \pm 5 \mathrm{~s}$, but there is observable signal over the interval $T_{100}=178 \pm 5 \mathrm{~s}$ with more than $70 \mathrm{~s}$ of very faint extended emission after the end of the $T_{90}$ time interval. Since XRT started its observation at full timing and spectral resolution in windowed timing (WT) mode at $T_{0}+97 \mathrm{~s}$, there is $\mathrm{a} \sim 80$ s overlap between the BAT and XRT observations.

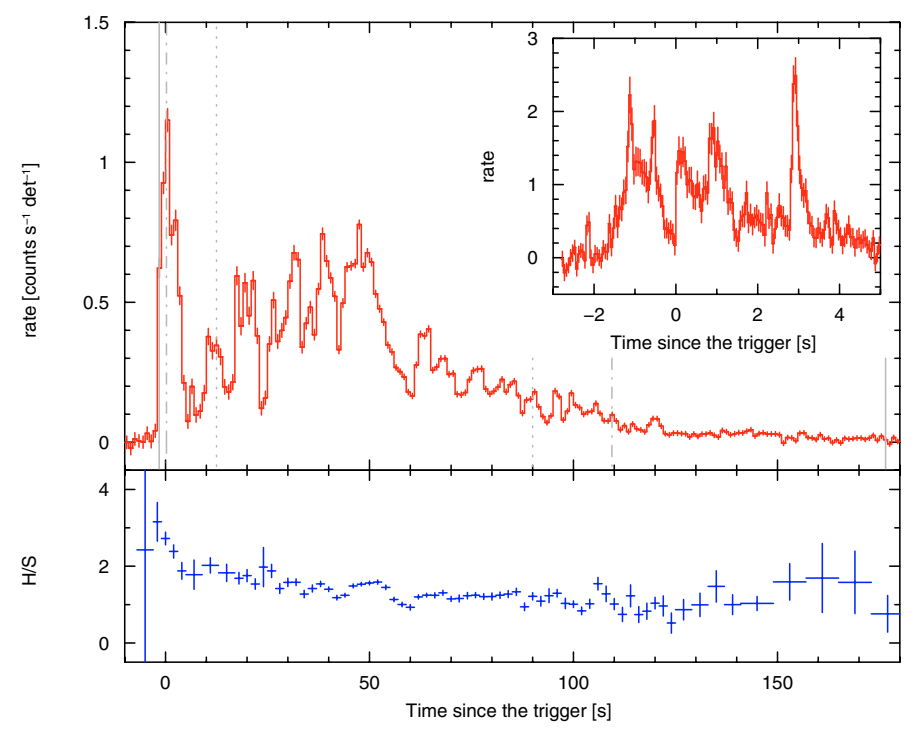

Fig. 2. In the upper panel the $15-150 \mathrm{keV}$ BAT light curve is shown at $1 \mathrm{~s}$ time resolution. Solid (dotted-dashed) vertical lines mark the $T_{100}$ $\left(T_{90}\right)$ time interval. Dotted vertical lines mark the start and the end of satellite slew. The inset shows the first group of peaks at $50 \mathrm{~ms}$ time resolution. Note that rate units are unchanged but peaks are higher because of the finer time resolution. The lower panel gives the hardness curve computed as the ratio of the count rate in the $25-150 \mathrm{keV}$ and in the $15-25 \mathrm{keV}$ band.

Table 3. Results of BAT time resolved spectral analysis.

\begin{tabular}{lccccc}
\hline \hline Spectrum & $\begin{array}{l}\text { Start } \\
\text { (s) }\end{array}$ & $\begin{array}{l}\text { End } \\
(\mathrm{s})\end{array}$ & $\Gamma$ & d.o.f. & $\chi_{\mathrm{r}}^{2}$ \\
\hline BAT & -2.83 & 176.5 & $2.13_{-0.04}^{+0.04}$ & 56 & 0.73 \\
\hline BAT-A & -2.83 & 5.62 & $1.63_{-0.07}^{+0.07}$ & 56 & 0.86 \\
BAT-B & 5.62 & 97.0 & $2.21_{-0.04}^{+0.04}$ & 56 & 0.73 \\
BAT-C & 97.0 & 176.5 & $2.37_{-0.13}^{+0.13}$ & 56 & 0.76 \\
\hline BAT-1 & -2.83 & -1.0 & $1.51_{-0.19}^{+0.20}$ & 56 & 0.93 \\
BAT-2 & -1.0 & 1.0 & $1.56_{-0.09}^{+0.09}$ & 56 & 1.31 \\
BAT-3 & 1.0 & 4.0 & $1.78_{-0.10}^{+0.10}$ & 56 & 0.75 \\
BAT-4 & 4.0 & 10.0 & $1.92_{-0.24}^{+0.25}$ & 56 & 0.83 \\
BAT-5 & 10.0 & 17.0 & $1.84_{-0.13}^{+0.13}$ & 56 & 0.85 \\
BAT-6 & 17.0 & 23.0 & $2.05_{-0.10}^{+0.11}$ & 56 & 1.01 \\
BAT-7 & 23.0 & 30.0 & $2.14_{-0.11}^{+0.11}$ & 56 & 0.71 \\
BAT-8 & 30.0 & 33.0 & $2.07_{-0.10}^{+0.10}$ & 56 & 0.62 \\
BAT-9 & 33.0 & 37.8 & $2.33_{-0.10}^{+0.10}$ & 56 & 0.88 \\
BAT-10 & 37.8 & 42.5 & $2.17_{-0.07}^{+0.07}$ & 56 & 1.02 \\
BAT-11 & 42.5 & 47.5 & $2.22_{-0.06}^{+0.06}$ & 56 & 0.92 \\
BAT-12 & 47.5 & 60.0 & $2.07_{-0.05}^{+0.05}$ & 56 & 1.03 \\
BAT-13 & 60.0 & 80.0 & $2.24_{-0.06}^{+0.06}$ & 56 & 0.79 \\
BAT-14 & 80.0 & 97.0 & $2.14_{-0.08}^{+0.08}$ & 56 & 0.75 \\
BAT-15 & 97.0 & 128.0 & $2.46_{-0.12}^{+0.12}$ & 56 & 1.09 \\
BAT-16 & 128.0 & 175.0 & $2.19_{-0.21}^{+0.22}$ & 56 & 0.66 \\
\hline & & & & & \\
\hline
\end{tabular}

BAT spectra were extracted over the $T_{100}$ time interval (from $T_{0}-1.55 \mathrm{~s}$ to $T_{0}+176.5 \mathrm{~s}$, first row in Table 3 ), over the time interval corresponding to the Konus-Wind observation of the first group of peaks of the burst (from $T_{0}-2.83 \mathrm{~s}$ to $T_{0}+5.62 \mathrm{~s}$ ), over the time interval of simultaneous BAT/XRT observation (from $T_{0}+97 \mathrm{~s}$ to $T_{0}+176.5 \mathrm{~s}$ ), and for the central part of the burst emission (from $T_{0}+5.62 \mathrm{~s}$ to $T_{0}+97 \mathrm{~s}$ ). The last three spectra are indicated in Table 3 (first column) as BAT-A, BAT-C and 


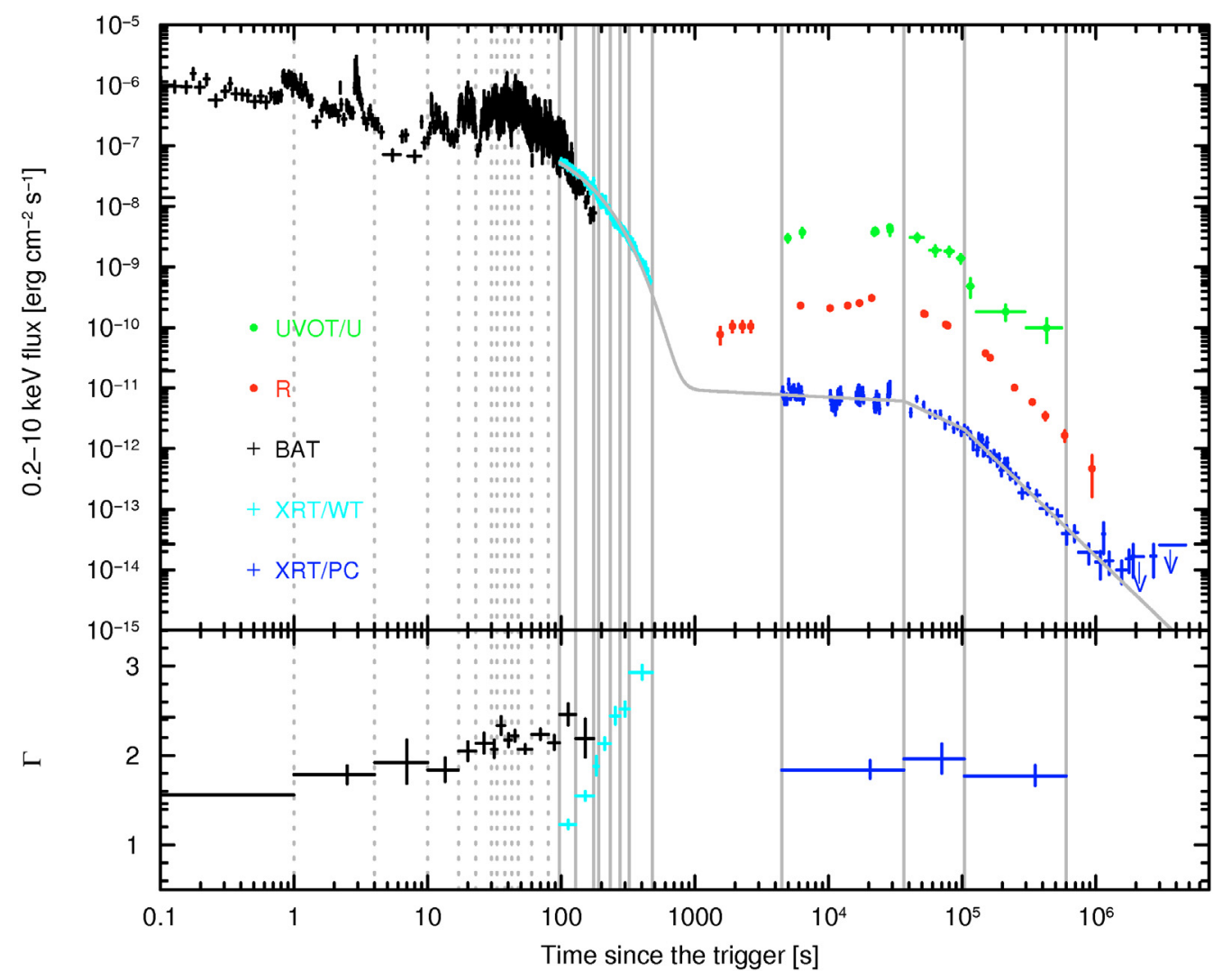

Fig. 3. Upper panel: the XRT light curve converted to flux in the $0.2-10 \mathrm{keV}$ energy range is shown together with the BAT light curve extrapolated to the same energy range. The joint best fit of the BAT and XRT spectra in the time interval 97-175 s has been used to calculate conversion factors for the BAT and XRT WT mode data. The best power-law fit of the average PC spectrum was used to convert XRT data in PC mode. The XRT light curve best fit model (solid line) is over-plotted on the data. The dashed vertical lines show the time intervals used for extraction of the BAT spectra (Table 3), while the solid vertical lines show the time intervals used for extraction of XRT spectra (corresponding to break times in the light curve during PC observation, i.e. $t>1000 \mathrm{~s}$ ). The $R$-band light curve (with the host galaxy contribution subtracted) is shown in arbitrary flux units for comparison. This light curve is mainly composed of VLT data (Della Valle et al. 2006) and complemented with other data available in the literature (Fynbo et al. 2006b; Gal-Yam et al. 2006; French et al. 2006). The $U$-band light curve by UVOT is also plotted in arbitrary flux units (with the possible host galaxy contribution subtracted). Lower panel: plot of the photon index of BAT and XRT spectra as a function of time (see Tables 3-5). Note that during the BAT and XRT overlap time interval, the BAT and XRT photon indices correspond to the high energy and low energy branches of a Band model (Band et al. 1993), respectively (see results of BAT and XRT joint fit in Table 8).

BAT-B, respectively. Moreover, BAT spectra were extracted over the 16 time intervals listed in Table 3 to allow for time resolved spectral analysis. These time intervals are shown by vertical dotted lines in Fig. 3.

Response matrices were generated with the task batdrmgen using the latest spectral redistribution matrices. For each spectrum, relevant keywords for response matrix generation were updated with the batupdatephakw task. For our spectral fitting (XSPEC v11.3.2) we considered the $14-150 \mathrm{keV}$ energy range and applied the latest energy-dependent systematic error vector provided by the CALDB distribution ${ }^{1}$.

\section{2. $X R T$}

The XRT data were first processed by the Swift Data Center at NASA/GSFC into Level 1 products (event lists). Then they were further processed with the XRTDAS (v1.8.0) software package, written by the ASI Science Data Center (ASDC) and distributed within FTOOLS to produce the final, cleaned event lists. In particular, we ran the task xrtpipeline (v0.10.3) applying calibration and standard filtering and screening criteria. For our analysis

${ }^{1}$ http://heasarc.gsfc .nasa.gov/docs/swift/analysis/ bat_digest.html we selected XRT grades $0-12$ and $0-2$ for photon counting (PC) and WT data, respectively (according to Swift nomenclature; Burrows et al. 2005; Hill et al. 2004). The X-ray counterpart was detected at the position $\mathrm{RA}_{\mathrm{J} 2000}=21^{\mathrm{h}} 23^{\mathrm{m}} 32^{\mathrm{s}} .00, \operatorname{Dec}_{\mathrm{J} 2000}=$ $-53^{\circ} 01^{\prime} 39^{\prime} .4$, with an estimated uncertainty of $33^{\prime \prime} 7$. This position was determined using the xrtcentroid task (v0.2.7) on the PC data in sequence 000 , that are not affected by pile-up, and it takes into account the correction for the misalignment between the telescope and the satellite optical axis. It is $51^{\prime \prime} .7$ from the refined BAT position.

\subsubsection{First orbit data}

During sequence 000 the count rate of the burst was high enough to cause pile-up in the WT mode data, that covered the entire first orbit XRT observation from $T_{0}+97$ to $T_{0}+480 \mathrm{~s}$. Therefore, to account for this effect, the WT data were extracted in a rectangular $40 \times 20$-pixel region with a $9 \times 20$-pixel region excluded from its centre. The size of the exclusion region was determined following the procedure illustrated in Romano et al. (2006): the analysis of the fraction of events at grade 0 for different sizes of the central hole in the extraction region saturates to a constant value for sizes greater than 10 pixels. To account for the background, WT events were also extracted within a rectangular box $(40 \times 20$ pixels $)$ far from background sources. 
Background subtracted WT light curves were extracted in the $0.2-1.0 \mathrm{keV}, 1.0-10 \mathrm{keV}$ and $0.2-10 \mathrm{keV}$ energy ranges (see Figs. 3 and 4). They were corrected for both the fraction of point spread function (PSF) lost due to the central hole in the extraction region and exposure variations within the extraction region. A vignetting correction was also properly applied.

Time-resolved spectral analysis of WT data was performed on 7 time intervals (see Table 4 and Fig. 3) selected according to the source brightness and requiring at least 2000 net counts each. The time resolved spectra were extracted from a rectangular $40 \times 20$-pixel region with a central rectangular region excluded. Different sizes of the excluded region were used according to the analysis of the fraction of events at grade 0 (see details in Table 4). Note that spectra WT-1 and WT-2 are simultaneous to spectra BAT-15 and BAT-16, respectively.

An average WT spectrum and a spectrum simultaneous to the tail of the BAT light curve (i.e. simultaneous to the BAT-C spectrum) were also extracted. The former is labeled as WT in the first column of Table 4, and the latter is labeled as WT- 0 . Both were extracted using the 10 pixels wide central hole to correctly account for the highest degree of pile-up at the beginning of the observation.

\subsubsection{Data taken after $1000 \mathrm{~s}$}

From the second orbit on, XRT observed in PC mode. For the PC data, which were never affected by pile-up throughout all the XRT observations, we extracted the source events in a circular region of 30 pixel radius up to segment 003 (i.e. $t \sim 556 \mathrm{ks}$ ). PC background data were also extracted in a source-free circular region (radius 40 pixels), and the background subtracted light curves (in the $0.2-1.0 \mathrm{keV}, 1.0-10 \mathrm{keV}$ and $0.2-10 \mathrm{keV}$ energy ranges) were corrected for the fraction of PSF lost, for timedependent exposure variations within the extraction region and for the vignetting effect.

For sequences from 004 to 037 the light curve points were calculated using the task sosta of the ximage package, which calculates vignetting- and PSF-corrected count rates within a specified box, and the background in a user-specified region. The background was estimated in the same region as the one used for the initial part of the light curve. Starting from sequence 006, data segments were merged until source detection with signal to noise ratio larger than 3 was attained (see Table 1). The last detection shown in Fig. 3 at $2.7 \mathrm{Ms}$, having a signal to noise ratio of 1.8 , may be a statistical fluctuation.

Three PC spectra were extracted according to different evolutionary stages in the light curve (see Sect. 3.1). They are listed in Table 5. Ancillary response files were generated with the task xrtmkarf within FTOOLS, and account for different extraction regions and PSF corrections. The basic files describing the XRT effective area used were the swxpc0to12_20010101v008.arf (for PC) and swxwt0to2_20010101v008.arf (for WT) from the latest distribution of the XRT Calibration Database (CALDB 2.4) maintained by HEASARC. We also used the latest spectral redistribution matrices in the Calibration Database, namely the file swxpc0to12_20010101v008.rmf for PC spectra and the file swxwtQto2_20010101v008.rmf for WT spectra.

\subsection{UVOT}

The first UVOT observation was a $97 \mathrm{~s}$ White finding chart taken in IMAGE\&EVENT mode starting $104 \mathrm{~s}$ after the BAT

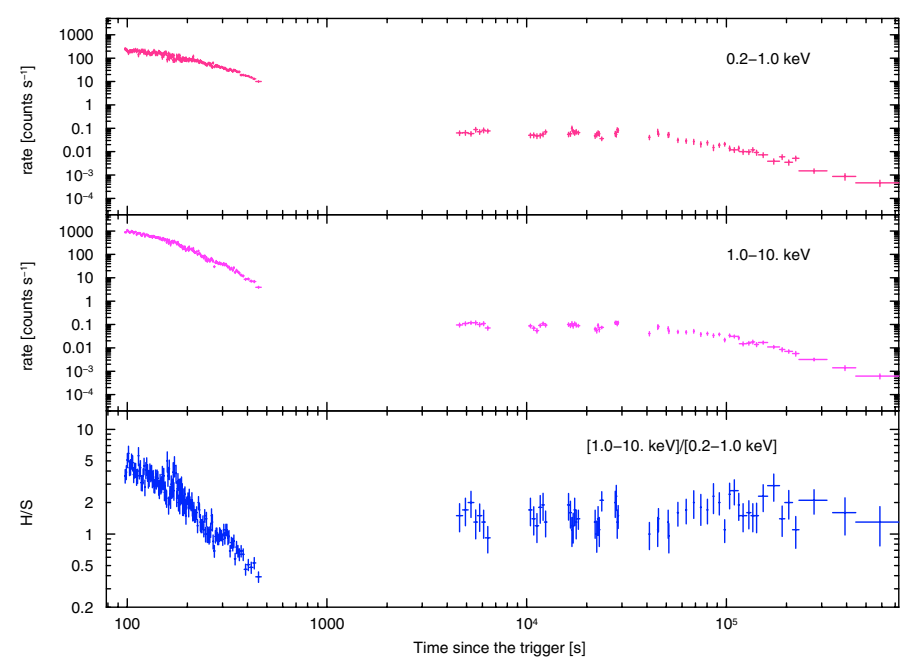

Fig. 4. XRT light curves in the $0.2-1.0 \mathrm{keV}$ (upper panel) and $1.0-10 \mathrm{keV}$ (middle panel) energy bands and hardness ratio curve (lower panel).

trigger (Holland 2006b). In this first observation the UVOT located the optical afterglow of GRB 060614 at $\mathrm{RA}_{\mathrm{J} 2000}=$ $21^{\mathrm{h}} 23^{\mathrm{m}} 32^{\mathrm{s}} .08, \operatorname{Dec}_{\mathrm{J} 2000}=-53^{\circ} 01^{\prime} 36^{\prime \prime} \cdot 2$ with a $90 \%$ confidence interval of 0.56 . This is $3^{\prime \prime} .3$ from the centre of the XRT error circle.

To extract UVOT light curves we performed aperture photometry on the UVOT exposures using a circular aperture with a radius of $2^{\prime \prime}$ centred on the UVOT position of the afterglow. A sky annulus of width 7'.5 and inner radius $27 . .5$ was used. This annulus includes a large number of sky pixels and is large enough to exclude the faint outer regions of the point-spread function of the afterglow. We performed aperture corrections to convert the $2^{\prime \prime}$ aperture photometry to the standard photometric apertures used to define the UVOT photometric zero points (6" for $U B V$ and $12^{\prime \prime}$ for the $U V W 1, U V M 2, U V W 2$, and White filters). Approximately between 5 and 7 isolated stars (depending on the filter) were used to compute aperture corrections for each exposure. The rms scatter in the aperture corrections for each source in a single exposure is typically $0.02 \mathrm{mag}$. The instrumental magnitudes were transformed to Vega magnitudes using the photometric zero points in the Swift/UVOT calibration database (CALDB). Colour terms have not been applied, but preliminary calibrations suggest that they are negligible for sources with typical afterglow colours. The adopted photometric zero points are $\mathrm{ZP}_{V}=17.88 \pm 0.09, \mathrm{ZP}_{B}=19.16 \pm 0.12, \mathrm{ZP}_{U}=$ $18.38 \pm 0.23, \mathrm{ZP}_{U V W 1}=17.69 \pm 0.02, \mathrm{ZP}_{U V M 2}=17.29 \pm 0.23$ $\mathrm{ZP}_{U V W 2}=17.77 \pm 0.02$, and $\mathrm{ZP}_{\text {White }}=19.78 \pm 0.02$.

Figure 5 shows the Swift-UVOT $U V W 2, U V M 2, U V W 2, U$, $B, V$ and White light curves, together with the $R$-band light curve of the afterglow mostly composed by VLT data (Della Valle et al. 2006) and complemented with points taken from the literature (Fynbo et al. 2006b; Gal-Yam et al. 2006; French et al. 2006). The flux densities are monochromatic fluxes for the central wavelength of each filter. They have been corrected for the Schlegel et al. (1998) Galactic extinction along the line of sight to GRB 060614 ( $\left.A_{V}=0.07 \mathrm{mag}\right)$.

The afterglow of GRB 060614 becomes brighter at optical and ultraviolet (UV) wavelengths until approximately eight hours after the BAT trigger and then fades. There is weak evidence for small-timescale fluctuations around the average light curve between approximately 3 and $28 \mathrm{~h}$. This may be due to energy injection during the first day after the burst; however, due 
Table 4. Results of XRT time-resolved spectral analysis: WT data.

\begin{tabular}{|c|c|c|c|c|c|c|c|c|}
\hline Spectrum & WT & WT-1 & WT-2 & WT-3 & WT-4 & WT-5 & WT-6 & WT-7 \\
\hline Time $^{a}$ & $97-480$ & $97-128$ & $128-175$ & $175-190$ & $190-233$ & $233-275$ & $275-322.5$ & $322.5-480$ \\
\hline Central hole $^{b}$ & 10 & 10 & 7 & 5 & 3 & 2 & 0 & 0 \\
\hline \multicolumn{9}{|c|}{ Power-law model } \\
\hline$N_{\mathrm{H}}^{c}$ & $3.6_{-0.8}^{+0.8}$ & $2.0_{-1.5}^{+2.0}$ & $7.0_{-2.0}^{+2.0}$ & $10.0_{-3.0}^{+4.0}$ & $9.3_{-1.9}^{+2.1}$ & $7.4_{-1.9}^{+2.2}$ & $5.0_{-1.1}^{+1.3}$ & $5.5_{-1.1}^{+1.2}$ \\
\hline$\Gamma$ & $1.61_{-0.03}^{+0.03}$ & $1.23_{-0.05}^{+0.05}$ & $1.55_{-0.05}^{+0.05}$ & $1.88_{-0.10}^{+0.11}$ & $2.13_{-0.07}^{+0.07}$ & $2.44_{-0.09}^{+0.10}$ & $2.52_{-0.08}^{+0.08}$ & $2.93_{-0.08}^{+0.08}$ \\
\hline d.o.f. & 386 & 185 & 243 & 69 & 160 & 96 & 119 & 125 \\
\hline$\chi_{\mathrm{r}}^{2}$ & 1.19 & 1.11 & 1.23 & 1.11 & 1.32 & 1.48 & 1.03 & 1.17 \\
\hline \multicolumn{9}{|c|}{ Power-law model with exponential cut off } \\
\hline$N_{\mathrm{H}}^{c}$ & $<0.9$ & $<0.6$ & $<1.0$ & $3.6_{-3.3}^{+4.4}$ & $<1.5$ & $<2.5$ & $1.7_{-1.2}^{+2.1}$ & $2.0_{-0.9}^{+2.0}$ \\
\hline$\Gamma$ & $1.19_{-0.03}^{+0.09}$ & $0.86_{-0.11}^{+0.11}$ & $0.89_{-0.09}^{+0.12}$ & $1.20_{-0.38}^{+0.40}$ & $1.07_{-0.08}^{+0.19}$ & $1.48_{-0.17}^{+0.29}$ & $1.86_{-0.22}^{+0.34}$ & $2.17_{-0.19}^{+0.38}$ \\
\hline$E_{\mathrm{p}}{ }^{d}$ & $5.3_{-0.8}^{+1.4}$ & $8.28_{-2.22}^{+4.00}$ & $4.91_{-0.99}^{+1.09}$ & $3.00_{-1.47}^{+4.38}$ & $2.21_{-0.44}^{+0.58}$ & $1.18_{-0.33}^{+0.58}$ & $0.42_{-0.13}^{+0.42}$ & $-{ }^{-0.19}$ \\
\hline d.o.f. & $385^{0.0}$ & 184 & 242 & 68 & $159^{-0.44}$ & 95 & 118 & 124 \\
\hline$\chi_{\mathrm{r}}^{2}$ & 1.04 & 0.97 & 1.05 & 1.01 & 0.97 & 1.22 & 0.92 & 1.03 \\
\hline \multicolumn{9}{|c|}{ Band model } \\
\hline$N_{\mathrm{H}}^{c}$ & $<0.4$ & $<0.6$ & $<0.7$ & $4.2_{-2.1}^{+2.5}$ & $<1.0$ & $<0.6$ & $<1.0$ & $<1.7$ \\
\hline$\alpha_{\text {Band }^{e}}$ & $-1.11_{-0.09}^{+0.09}$ & $-0.86_{-0.11}^{+0.11}$ & $-0.78_{-0.13}^{+0.16}$ & -1.20 & -1.07 & -1.00 & $-1.17_{-0.17}^{+0.07}$ & $-1.63_{-0.15}^{+0.11}$ \\
\hline$\beta_{\text {Band }^{e}}^{e}$ & $<-1.8^{-0.09}$ & $<-4.5^{-0.11}$ & $<-1.7^{-0.15}$ & $<-1.9$ & $<-2.47$ & $-2.62_{-0.20}^{+0.15}$ & $-2.52^{-0.11}$ & $-2.93^{-0.10}$ \\
\hline$E_{\mathrm{p}}{ }^{d}$ & $4.2_{-0.6}^{+1.43}$ & $8.34_{-1.36}^{+1.47}$ & $4.04_{-1.25}^{+1.60}$ & $2.63_{-0.55}^{+0.75}$ & $2.17_{-0.20}^{+0.14}$ & $1.11_{-0.10}^{+0.10}$ & $<0.91$ & $<0.64$ \\
\hline d.o.f. & 384 & 183 & 241 & 68 & $159^{-0.20}$ & $95^{-0.10}$ & 118 & 124 \\
\hline$\chi_{\mathrm{r}}^{2}$ & 1.03 & 0.98 & 1.03 & 1.00 & 0.96 & 1.14 & 0.95 & 1.03 \\
\hline \multicolumn{9}{|c|}{ Power-law model plus blackbody } \\
\hline$N_{\mathrm{H}}^{c}$ & $<1.0$ & $<0.9$ & $<1.6$ & $<7.4$ & $<3.5$ & $<2.0$ & $2.9_{-1.7}^{+1.7}$ & $2.4_{-1.4}^{+1.5}$ \\
\hline$\Gamma$ & $0.55_{-0.06}^{+0.08}$ & $1.23_{-0.07}^{+0.09}$ & $1.34_{-0.05}^{+0.09}$ & $1.62_{-0.26}^{+0.23}$ & $1.90_{-0.14}^{+0.13}$ & $2.05_{-0.06}^{+0.15}$ & $2.44_{-0.15}^{+0.14}$ & $2.77_{-0.13}^{+0.13}$ \\
\hline$k T_{\mathrm{bb}}^{f}$ & $1.94_{-0.49}^{+0.40}$ & $0.89_{-0.15}^{+0.16}$ & $0.60_{-0.06}^{+0.07}$ & $0.465_{-0.069}^{+0.120}$ & $0.47_{-0.05}^{+0.06}$ & $0.31_{-0.03}^{+0.036}$ & $0.35_{-0.07}^{+0.08}$ & $0.25_{-0.03}^{+0.04}$ \\
\hline$R_{\mathrm{bb}}^{g}$ & $1.50_{-0.05}^{+0.06}$ & $2.1_{-0.5}^{+0.7}$ & $3.4_{-0.7}^{+0.6}$ & $4.2_{-1.9}^{+1.9}$ & $3.5_{-0.8}^{+1.0}$ & $5.7_{-1.4}^{+1.1}$ & $2.5_{-1.1}^{+1.6}$ & $3.5_{-1.2}^{+1.4}$ \\
\hline d.o.f. & 384 & 183 & 241 & 67 & 158 & 94 & 117 & 123 \\
\hline$\chi_{\mathrm{r}}^{2}$ & 1.05 & 0.95 & 1.04 & 0.96 & 1.01 & 1.19 & 0.91 & 1.00 \\
\hline$F_{\mathrm{bb}} / F^{h}$ & 0.10 & 0.16 & 0.17 & 0.18 & 0.22 & 0.23 & 0.10 & 0.12 \\
\hline Ftest $^{i}$ & $1.9 \times 10^{-11}$ & $2.4 \times 10^{-7}$ & $7.9 \times 10^{-10}$ & $1.8 \times 10^{-20}$ & $1.5 \times 10^{-10}$ & $1.0 \times 10^{-5}$ & $3.3 \times 10^{-4}$ & $3.1 \times 10^{-5}$ \\
\hline
\end{tabular}

${ }^{a}$ Start and stop times of the time interval in seconds.

${ }^{b}$ Width in pixels of the central box (20 pixel thick) that we excluded from the $40 \times 20$ pixel extraction region to account for the pile-up effect.

${ }^{c}$ Extragalactic absorption column in units of $10^{20} \mathrm{~cm}^{-2}$. The model included both a wabs component accounting for Galactic absorption (i.e. with $N_{\mathrm{H}}$ fixed to $3 \times 10^{20} \mathrm{~cm}^{-2}$; Dickey \& Lockman 1990) and a zwabs component with redshift fixed to $z=0.125$ and free $N_{\mathrm{H}}$ parameter to account for extragalactic absorption.

${ }^{d}$ Peak energy in units of keV. It is related to the cut-off energy $E_{\text {cut }}$ through the equation $E_{\mathrm{p}}=(2-\Gamma) E_{\text {cut }}$ for the cut-off power law model (provided that $\Gamma<2)$ and $E_{\mathrm{p}}=\left(2+\alpha_{\text {Band }}\right) E_{\text {cut }}$ for the Band model.

${ }^{e} \alpha_{\text {Band }}$ and $\beta_{\text {Band }}$ are the low and high energy indices of the Band model, respectively.

${ }^{f}$ Blackbody temperature in units of $\mathrm{keV}$.

${ }^{g}$ Blackbody radius in units of $10^{11} \mathrm{~cm}$.

${ }^{h}$ Unabsorbed flux of the blackbody component in the $0.2-10 \mathrm{keV}$ range $\left(F_{\mathrm{bb}}\right)$ over the total unabsorbed flux $(F)$.

${ }^{i}$ Chance probability of improvement of the fit adding a blackbody component to the absorbed power-law.

Notes:

i) The first column of the table corresponds to the integrated WT spectrum.

ii) Parameter values are reported without errors when frozen in the fitting procedure.

Table 5. Results of XRT time-resolved analysis: PC data.

\begin{tabular}{lllllll}
\hline \hline Spectrum & $\begin{array}{l}\text { Start time } \\
(\mathrm{s})\end{array}$ & $\begin{array}{l}\text { End time } \\
(\mathrm{s})\end{array}$ & $\begin{array}{l}N_{\mathrm{H}}{ }^{a} \\
\left(10^{20} \mathrm{~cm}^{-2}\right)\end{array}$ & $\Gamma$ & d.o.f. & $\chi_{\mathrm{r}}^{2}$ \\
\hline PC-1 & 4448.6 & 36575.4 & $<1.9$ & $1.84_{-0.095}^{+0.105}$ & 66 & 1.08 \\
PC-2 & 36575.4 & 103701.8 & $<3.9$ & $1.96_{-0.16}^{+0.17}$ & 25 & 0.96 \\
PC-3 & 103701.8 & 598638.2 & $<2.9$ & $1.77_{-0.11}^{+0.12}$ & 51 & 0.83 \\
\hline PC & 4448.6 & 598638.2 & $1.5_{-1.2}^{+1.2}$ & $1.84_{-0.08}^{+0.08}$ & 146 & 0.99 \\
\hline
\end{tabular}

${ }^{a}$ Extragalactic absorption column. The model included both a wabs component accounting for Galactic absorption (i.e. with $N_{\mathrm{H}}$ fixed to $3 \times$ $10^{20} \mathrm{~cm}^{-2}$; Dickey \& Lockman 1990) and a zwabs component with redshift fixed to $z=0.125$ and free $N_{\mathrm{H}}$ parameter to account for extragalactic absorption. 


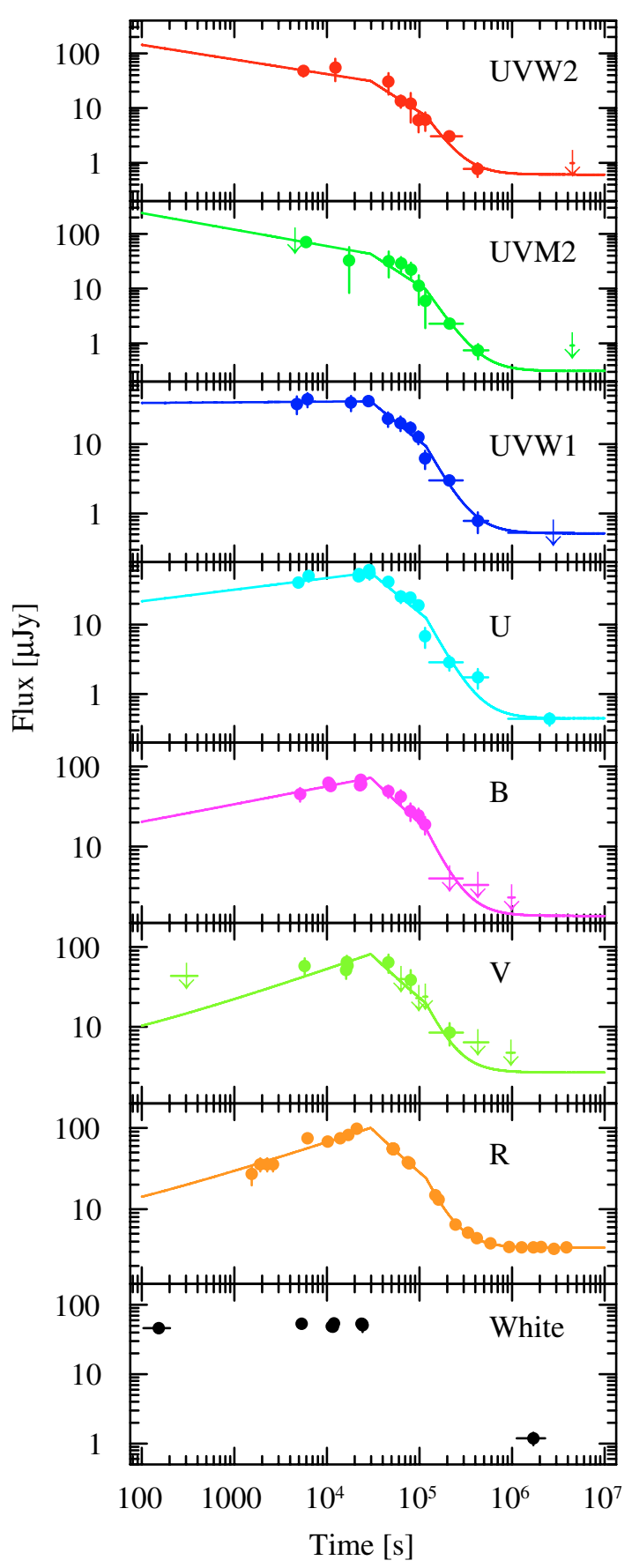

Fig. 5. UVOT light curves in different filters and $R$-band light curve obtained from VLT data (Della Valle et al. 2006) and other data in the literature (Fynbo et al. 2006b; Gal-Yam et al. 2006; French et al. 2006). All light curves are corrected for the Galactic extinction along the line of sight to GRB 060614. The best fit model discussed in the text is represented by solid lines.

to the photometric uncertainty in the data, and the inherent difficulties with co-adding images of a variable source, it is not clear if these fluctuations are real. We note that the $R$-band light curve (as observed by ground based telescopes) shows small-timescale variability as well. The $U$ and $U V M 2$ light curves show weak evidence for change in the decay rate at approximately $28 \mathrm{~h}$ after the BAT trigger.

The UVOT $U$-band detection above the power law fit to the light curve at $\sim 500 \mathrm{ks}$ was previously interpreted as a possible SN (Holland 2006a; Brown 2006). In view of the extremely low limits on a SN contribution obtained by groundbased and HST observations (Della Valle et al. 2006; Fynbo et al. 2006b; Gal-Yam et al. 2006), we now interpret this excess as a statistical fluctuation. The shape of the excess is hard to determine due to the faintness of the afterglow and the subsequent exposure time (spanning days) needed to detect it, and is dependent on how the power-law is fitted to the earlier data and how the late time data are co-added. We present here the most conservative version, consisting of a single detection about five days after the burst which lies above the power-law fit, with the later data co-added together to determine the host galaxy brightness.

\section{Data analysis}

\subsection{The BAT and XRT light curves}

The XRT light curve in $0.2-10 \mathrm{keV}$ flux units is shown in Fig. 3 together with the BAT light curve extrapolated to the XRT energy band. Both light curves have been converted to flux using the best fit model parameters of the joint fit of the BAT and XRT spectra extracted from the BAT/XRT overlap time interval 97-176.5 s (spectra BAT-C and WT-0, respectively; see Sect. 3.3). Note that the best fit model to the BAT and XRT spectra in the overlap time interval, as presented in Sect. 3.3, is a Band model (Band et al. 1993) with peak energy $E_{\mathrm{p}} \sim 8 \mathrm{keV}$, and the extrapolation of the BAT light curve to the XRT energy range according to this model naturally leads to a very good match between the tail of the BAT and the start of the XRT light curve, but it underestimates slightly the $0.2-10 \mathrm{keV}$ emission of the burst before the start of the XRT observation, when the peak energy of the spectrum was certainly higher (as high as $\sim 300 \mathrm{keV}$ during the first episode in BAT). However, a timedependent conversion of the BAT light curve to the XRT energy range cannot be performed because of our ignorance about the exact $E_{\mathrm{p}}$ value after the initial group of BAT peaks and before the start of the XRT observation.

The XRT/WT light curve can be modeled as an exponential decay, while the XRT/PC light curve is well fitted by a doubly broken power law model. Results of the light curve fit are presented in Table 6 . Note that points at $t>1.8 \mathrm{Ms}$ slightly deviate from the best fit model and suggest a late re-brightening or a flattening of the X-ray afterglow. However, the last detection at 2.7 Ms is only a $1.8-\sigma$ detection, and the following upper limit tells us that at the end the source faded below the XRT sensitivity limit.

A fit with an additional constant (to account for the late flattening seen in the light curve) gives a slightly steeper slope $\alpha_{\mathrm{C}}=$ $2.2 \pm 0.1$, and a constant flux of $\sim(8 \pm 4) \times 10^{-15} \mathrm{erg} \mathrm{cm}^{-2} \mathrm{~s}^{-1}$. This would correspond to an X-ray luminosity of about $3 \times$ $10^{41} \mathrm{erg} \mathrm{s}^{-1}$. This value is by far larger than what can be provided by the ongoing star formation activity: using the star formation rate $1.3 \times 10^{-2} M_{\odot} \mathrm{yr}^{-1}$ (Della Valle et al. 2006) and the conversion factor by Grimm et al. (2003), this could contribute $\lesssim 10^{36} \mathrm{erg} \mathrm{s}^{-1}$. The X-ray luminosity could however be due to a small AGN. A further study of the optical spectrum of the galaxy mighty clarify this issue. We note however that Gal-Yam et al. (2006) show that the light profile of the galaxy is well fitted by an exponential disk model, without the need of a nuclear component. Therefore, the apparent flattening of the late X-ray light curve may also be a statistical fluctuation.

The $0.2-1.0 \mathrm{keV}$ and 1.0-10 keV XRT light curves are shown in the first two panels of Fig. 4. They have been rebinned so as to have at least 100 counts per bin in each energy band 
Table 6. GRB 060614 X-ray light curve best fit parameters.

\begin{tabular}{llc}
\hline \hline Parameter & & Value \\
\hline$\tau$ & $(\mathrm{s})$ & $75.7 \pm 0.4$ \\
$\alpha_{\mathrm{A}}$ & & $0.11 \pm 0.05$ \\
$T_{\mathrm{b}, 1}$ & $\left(10^{3} \mathrm{~s}\right)$ & $36.6 \pm 2.4$ \\
$\alpha_{\mathrm{B}}$ & & $1.03 \pm 0.02$ \\
$T_{\mathrm{b}, 2}$ & $\left(10^{3} \mathrm{~s}\right)$ & $103.7 \pm 22.0$ \\
$\alpha_{\mathrm{C}}$ & & $2.13 \pm 0.07$ \\
$\chi^{2}$ (d.o.f.) & $(\mathrm{WT})$ & $319(183)$ \\
$\chi^{2}$ (d.o.f.) & $(\mathrm{PC})$ & $119(97)$ \\
\hline
\end{tabular}

Note. The model used to fit the XRT light curve consisted of an exponential decay law $(\propto \exp -t / \tau)$ plus a doubly-broken power-law. The parameters $\alpha_{\mathrm{A}}, \alpha_{\mathrm{B}}$, and $\alpha_{\mathrm{C}}$ are the decay slopes for the distinct phases of the afterglow. $T_{\mathrm{b}, 1}$ and $T_{\mathrm{b}, 2}$ are the epochs at which the decay slope changes, measured from the GRB onset. Contributions to the $\chi^{2}$ of the WT and PC datasets have been indicated.

Table 7. GRB 060614 optical light curve best fit parameters.

\begin{tabular}{lllc}
\hline \hline Parameter & Filter & & Value \\
\hline$h$ & $U V W 2$ & (mag) & $23.25 \pm 0.54$ \\
$h$ & $U V M 2$ & (mag) & $24.1 \pm 1.0$ \\
$h$ & $U V W 1$ & (mag) & $23.5 \pm 0.7$ \\
$h$ & $U$ & (mag) & $23.8 \pm 0.3$ \\
$h$ & $B$ & (mag) & 23.73 \\
$h$ & $V$ & (mag) & 22.75 \\
$h$ & $R$ & (mag) & 22.46 \\
$\alpha_{\mathrm{A}}$ & $U V W 2$ & & $0.27 \pm 0.26$ \\
$\alpha_{\mathrm{A}}$ & $U V M 2$ & & $0.30 \pm 0.26$ \\
$\alpha_{\mathrm{A}}$ & $U V W 1$ & & $-0.01 \pm 0.20$ \\
$\alpha_{\mathrm{A}}$ & $U$ & & $-0.17 \pm 0.14$ \\
$\alpha_{\mathrm{A}}$ & $B$ & & $-0.23 \pm 0.15$ \\
$\alpha_{\mathrm{A}}$ & $V$ & & $-0.41 \pm 0.55$ \\
$\alpha_{\mathrm{A}}$ & $R$ & & $-0.38 \pm 0.23$ \\
$T_{\mathrm{b}, 1}$ & all & $\left(10^{3} \mathrm{~s}\right)$ & $29.7 \pm 4.4$ \\
$\alpha_{\mathrm{B}}$ & all & & $1.11 \pm 0.05$ \\
$T_{\mathrm{b}, 2}$ & all & $\left(10^{3} \mathrm{~s}\right)$ & $117.2 \pm 4.4$ \\
$\alpha_{\mathrm{C}}$ & all & & $2.44 \pm 0.08$ \\
$\chi^{2}$ (d.o.f.) & & & $68.815(74)$ \\
\hline
\end{tabular}

Note. The UVOT light curves and the $R$-band light curve of the GRB 060614 afterglow were fitted simultaneously with a constant plus a doubly-broken power law model. The parameter $h$ represents the constant value of the host galaxy contribution to the total observed flux. The $h$ values are not corrected for any extinction. The parameters $\alpha_{\mathrm{A}}$, $\alpha_{\mathrm{B}}$, and $\alpha_{\mathrm{C}}$ are the decay slopes for the distinct phases of the afterglow. $T_{\mathrm{b}, 1}$ and $T_{\mathrm{b}, 2}$ are the epochs at which the decay slope changes, measured from the GRB onset. During the fit only $h, \alpha_{\mathrm{A}}$ and the normalizations were allowed to be filter dependent.

during the first orbit (WT data) and at least 20 counts per bin in each energy band afterwards (PC data). A hardness ratio curve was computed and it is shown in the third panel. A strong hard to soft evolution is visible in the first $400 \mathrm{~s}$ of data. This is likely due to the passage of the peak energy across the XRT band (see Sect. 3.3).

Note that portions of smooth bumps are visible during the nearly flat light curve segment from 4 to $\sim 30 \mathrm{ks}$ and the hardness curve tracks them, although the hardness variations are small. Then, the light curve shows a hardening trend up to the time of the second break $T_{\mathrm{b}, 2} \sim 104 \mathrm{ks}$, and an irregular behaviour with oscillating hardness later on. However, the hardness curve at $t>4 \mathrm{ks}$ is well fitted by a constant with best fit value $1.42 \pm$ 0.05 ( $\chi^{2}=44.52$, with 56 d.o.f.).

\subsection{The UVOT light curves}

The Swift-UVOT $U V W 2, U V M 2, U V W 2, U, B, V$ and White light curves shown in Fig. 5 are too sparse for a detailed filterdependent fit. On the other hand, the $R$ band light curve of the afterglow obtained using data from the literature (Della Valle et al. 2006; Fynbo et al. 2006b; Gal-Yam et al. 2006; French et al. 2006), shown in the seventh panel from the top in Fig. 5 can be well fitted by a constant (accounting for the host galaxy emission) plus a doubly broken power law. We performed a joint fit to the UVOT and $R$-band light curves using the same model, with filter-dependent initial slope $\left(\alpha_{\mathrm{A}}\right)$, host galaxy contribution $(h)$, and normalization. The values of $h$ for the filters $B, V$ and $R$ were fixed to measured values: $B=23.73 \pm 0.13, V=22.75 \pm 0.05$, and $R=22.46 \pm 0.01$ (Della Valle et al. 2006). The White filter UVOT light curve, shown in the bottom panel of Fig. 5, does not sample uniformly the observation time interval and has not been included in the fit. The best fit parameters are listed in Table 7.

We find that the behaviour of all our optical and ultraviolet light curves is consistent with a first break at $T_{\mathrm{b}, 1}=29.7 \pm 4.4 \mathrm{ks}$, an after-break decay with slope $\alpha_{\mathrm{B}}=1.11 \pm 0.05$, a second break at $T_{\mathrm{b}, 2}=104 \pm 22.0 \mathrm{ks}$, and a final decay with slope $\alpha_{\mathrm{C}}=2.44 \pm$ 0.08 . The decay slope before the first break $\left(\alpha_{\mathrm{A}}\right)$ has a well defined trend with wavelength (decreasing from the ultraviolet to the optical), although errors are quite large. We note that, on the contrary, the light curve in the White filter is constant up to $30 \mathrm{ks}$.

The best fit values of the host galaxy contributions $h$ in the ultraviolet filters ( $U V W 2, U V M 2, U V W 1, U)$ are roughly consistent with those of a dwarf star-forming galaxy.

\subsection{BAT and XRT spectral analysis}

All BAT spectra are best fitted by single power law models. Results are shown in Table 3 . The BAT average spectrum of the burst has a photon index $\Gamma=2.13 \pm 0.04$, corresponding to a $15-150 \mathrm{keV}$ fluence of $2.2 \times 10^{-5} \mathrm{erg} \mathrm{cm}^{-2}$. A fit with a Band model (Band et al. 1993) with low energy in$\operatorname{dex} \alpha_{\text {Band }}$ fixed to -1 does not represent an improvement, but allows us to set an upper limit to the average peak energy of $E_{\mathrm{p}} \lesssim 24 \mathrm{keV}$ at the $90 \%$ confidence level. The BAT spectrum of the first episode of peaks (spectrum BAT-A in Table 3 ) has a photon index of $1.63_{-0.07}^{+0.07}$, consistent with the Konus-Wind low energy index $1.57_{-0.14}^{+0.12}$ (Golenetskii et al. 2006). The average spectrum of the following five broad peaks (spectrum BAT-B) is softer $(\Gamma=2.2 \pm 0.04)$. The BAT spectrum of the tail of the prompt emission (spectrum BAT-C) has a similar photon index, $\Gamma=2.37 \pm 0.13$. The $15-150 \mathrm{keV}$ fluence in the initial group of peaks is $3.4 \times 10^{-6} \mathrm{erg} \mathrm{cm}^{-2}$, while the rest of the burst provides a fluence of $1.9 \times 10^{-5} \mathrm{erg} \mathrm{cm}^{-2}$. The softening of the BAT emission is well represented in the lower panel of Fig. 3, where the best fit photon indices of the 16 BAT spectra are plotted as a function of time.

The XRT WT spectra have also been modeled with an absorbed single power law (see Table 4, top panel). All the fits were done in the $0.3-10 \mathrm{keV}$ energy range excluding channels between 0.45 and $0.55 \mathrm{keV}$ where an instrumental artefact commonly appears in XRT spectra ${ }^{2}$. Two absorption components were included in the model: a Galactic absorption component, fixed to the expected value $N_{\mathrm{H}}^{\mathrm{G}}=3 \times 10^{20} \mathrm{~cm}^{-2}$ according to

\footnotetext{
2 This artefact is probably caused either by a time-dependent energy offset, or by a problem in the detector response matrix, and is under active investigation by the XRT instrument team; see details in http://www.swift.ac.uk/xrt_bias.pdf.
} 
Table 8. Results of BAT and XRT joint spectral analysis.

\begin{tabular}{|c|c|c|c|}
\hline BAT Spectrum & BAT-C & BAT-15 & BAT-16 \\
\hline XRT Spectrum & WT-0 & WT-1 & WT-2 \\
\hline Time $^{a}$ & $97-176.5$ & $97-128$ & $128-175$ \\
\hline Central hole ${ }^{b}$ & 10 & 10 & 7 \\
\hline \multicolumn{4}{|c|}{ Band model } \\
\hline$N_{\mathrm{H}}{ }^{c}$ & $<0.7$ & $<0.6$ & $<0.9$ \\
\hline$\alpha_{\text {Band }}^{d}$ & $-0.89_{-0.08}^{+0.05}$ & $-0.86_{-0.11}^{+0.05}$ & $-0.84_{-0.13}^{+0.07}$ \\
\hline$\beta_{\text {Band }} d$ & $\begin{array}{r}-2.38_{-0.09}^{+0.07} \\
-\end{array}$ & $-2.46_{-0.06}^{+0.11}$ & $-2.10_{-0.17}^{+0.13}$ \\
\hline$E_{\mathrm{p}}{ }^{e}$ & $6.1_{-0.7}^{+1.3}$ & $8.3_{-1.2}^{+2.5 .06}$ & $4.5_{-1.1}^{+1.0 .17}$ \\
\hline (d.o.f.) & 359 & 240 & 298 \\
\hline$\chi_{r}^{2}$ & 0.99 & 1.00 & 0.97 \\
\hline \multicolumn{4}{|c|}{ Power-law model plus blackbody } \\
\hline$N_{\mathrm{H}}^{c}$ & $13.3_{-2.5}^{+2.9}$ & $10.9_{-3.1}^{+3.8}$ & $3.0_{-0.8}^{+6.0}$ \\
\hline$\Gamma$ & $2.1_{-0.1}^{+0.1}$ & $2.3_{-0.1}^{+0.1 .1}$ & $1.5_{-0.1}^{+0.8}$ \\
\hline$k T_{\mathrm{bb}}^{f}$ & $1.29_{-0.08}^{+0.09}$ & $1.31_{-0.09}^{+0.09}$ & $0.7_{-0.1}^{+0.1}$ \\
\hline$R_{\mathrm{bb}}^{g}$ & $1.4_{-0.2}^{+0.200}$ & $1.9_{-0.3}^{+0.3}$ & $2.2_{-0.7}^{+0.1}$ \\
\hline d.o.f. & $359^{-0.2}$ & $240^{-0.5}$ & $298^{-0.7}$ \\
\hline$\chi_{r}^{2}$ & 1.27 & 1.23 & 1.10 \\
\hline
\end{tabular}

${ }^{a}$ Start and stop times of the time interval in seconds. ${ }^{b}$ Length in pixels of the central box ( 20 pixel thick) that we excluded from the $40 \times 20$ pixel extraction region to account for the pile-up effect in XRT/WT data. ${ }^{c}$ Extragalactic absorption column in units $10^{20} \mathrm{~cm}^{-2}$. The model included both a wabs component accounting for Galactic absorption (i.e. with $N_{\mathrm{H}}$ fixed to $3 \times 10^{20} \mathrm{~cm}^{-2}$; Dickey \& Lockman 1990) and a zwabs component with redshift fixed to $z=0.125$ and free $N_{\mathrm{H}}$ parameter to account for extragalactic absorption. ${ }^{d} \alpha_{\text {Band }}$ and $\beta_{\text {Band }}$ are the low and high energy index of the Band model, respectively. ${ }^{e}$ Peak energy of the Band model in units of keV. ${ }^{f}$ Black body temperature in units of keV. ${ }^{g}$ Black body radius in units of $10^{11} \mathrm{~cm}$.

Note. The first column in the table corresponds to the BAT and XRT/WT spectra integrated over the whole BAT/XRT overlap time interval.

Dickey \& Lockman (1990), and an absorption component intrinsic to the host which was left free to vary. The simple power law fits implied intrinsic absorption at the level of $\sim 5 \times 10^{20} \mathrm{~cm}^{-2}$ in all of the XRT/WT spectra but showed an unphysical trend in the $N_{\mathrm{H}}$ consisting of a rise followed by a decay. Moreover, the reduced $\chi^{2}$ of the fits are marginally acceptable and careful inspection of the fit residuals put in evidence systematic trends below $2 \mathrm{keV}$ that suggest the presence of curvature in the spectra.

To account for the curvature of the WT spectra we fit them also with $i$ ) an absorbed cut-off power law model, $i i$ ) an absorbed Band model (Band et al. 1993), and iii) an absorbed power law model plus a blackbody component. The choice of the first two models is justified by the possibility that the early XRT light curve is the tail of the prompt emission, which usually has a Band spectrum (the Band spectrum can be approximated by a cut-off power law over a limited energy band). Model iii) was suggested by recent results obtained by Campana et al. (2006a) on GRB 060218 and Grupe et al. (2007) on GRB 060729. Best fit parameters of all XRT/WT spectra with these three models are shown in Table 4. All the three models gave us a significant improvement in the fit and are statistically equivalent. They give total reduced $\chi^{2}$ (calculated over the WT-1 to WT-7 spectra) of 1.02 (990 d.o.f), 1.01 (988 d.o.f.), and 1.01 (983 d.o.f.), respectively. They also constrain intrinsic absorption to the level of a few $\times 10^{20} \mathrm{~cm}^{-2}$ or less. In the model (iii) fits, the temperature of the blackbody component $k T_{\mathrm{bb}}$ smoothly decreases from 0.8 to $0.2 \mathrm{keV}$, while the photon index $\Gamma$ grows from 1.3

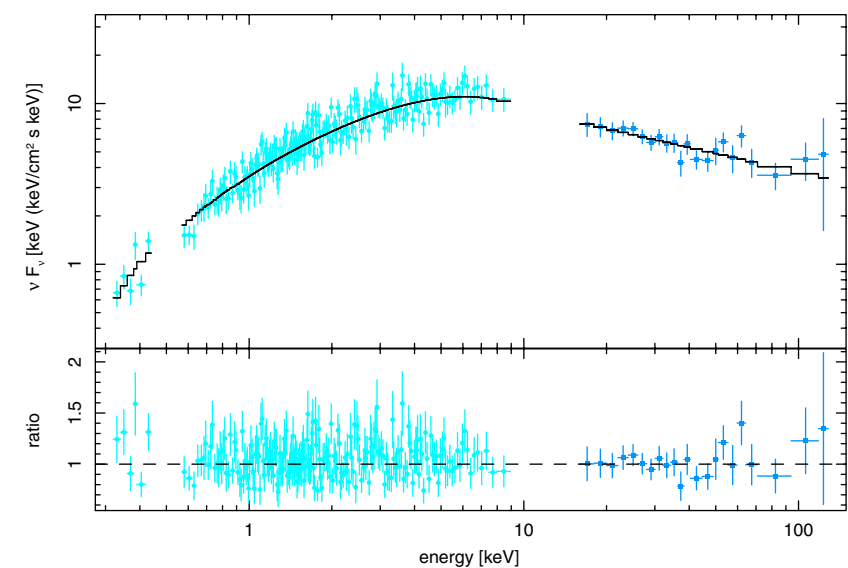

Fig. 6. Joint fit of the BAT and XRT spectra in the BAT/XRT overlap time interval. The best fit model is an absorbed Band model with peak energy $\sim 8 \mathrm{keV}$. See Table 8.

to 2.8 . The radius of the emitting region is of the order of $10^{11} \mathrm{~cm}$ and seems to peak at $\sim T_{0}+250 \mathrm{~s}$, as does the fraction of total flux contributed by the blackbody component. The fits with models $i$ ) and $i$ ), on the other hand, give us the alternative picture of a smooth peak energy decrease from $\sim 8 \mathrm{keV}$ to a value below the XRT energy range at about $\sim T_{0}+250 \mathrm{~s}$.

A clue to the true nature of the spectrum is found by a simultaneous fit to the BAT and XRT spectra during the overlap time when both instruments were detecting the burst (spectral pairs BAT-C and WT-0; BAT-15 and WT-1; BAT-16 and WT-2). When fitted with power laws, the BAT and XRT spectra show substantially different photon indices during these intervals, implying the existence of a break in the broad band spectrum. A joint fit of these pairs of spectra was therefore performed with an absorbed Band model. Results are shown in Table 8 (top panel) and in Fig. 6. A peak energy $E_{\mathrm{p}}$ of $8 \mathrm{keV}$ is detected during the BAT/XRT overlap (Fig. 6). A description of the joint BAT/XRT spectra with an absorbed power law model plus a blackbody is statistically less successful and requires an absorption column ten times higher than all previous fits (bottom panel of Table 8). This argues against the presence of a blackbody component in the early XRT spectra, though we cannot rule it out after $176 \mathrm{~s}$ post-burst.

The PC spectra (starting after $4 \mathrm{ks}$ from the trigger) are well fitted by a single power law model and did not show evidence of intrinsic absorption. Results are in Table 5. The low absorption values are consistent with those found for the WT data fit to intrinsically curved spectral models, adding additional evidence that the power law fits to the WT data are inappropriate. No significant spectral variation is seen throughout the XRT/PC observation. The three spectra extracted, corresponding to the three decay phases observed after $4 \mathrm{ks}$, show photon indices consistent with the average value of $1.85 \pm 0.12$.

\subsection{X-ray/optical spectral energy distributions}

The spectral energy distribution (SED) of the afterglow from optical to X-rays have been computed at several different times using the best fit models of the UVOT and VLT light curves presented in Sect. 3.2, the best fit model of the XRT light curve (Sect. 3.1) and the average XRT spectrum of the PC observation (Sect. 3.3 and Table 5). We selected the following times as representative of the afterglow evolutionary stages: 10, 30, 60 and $150 \mathrm{ks}$, i.e. a time before the first break/peak, the time of the 

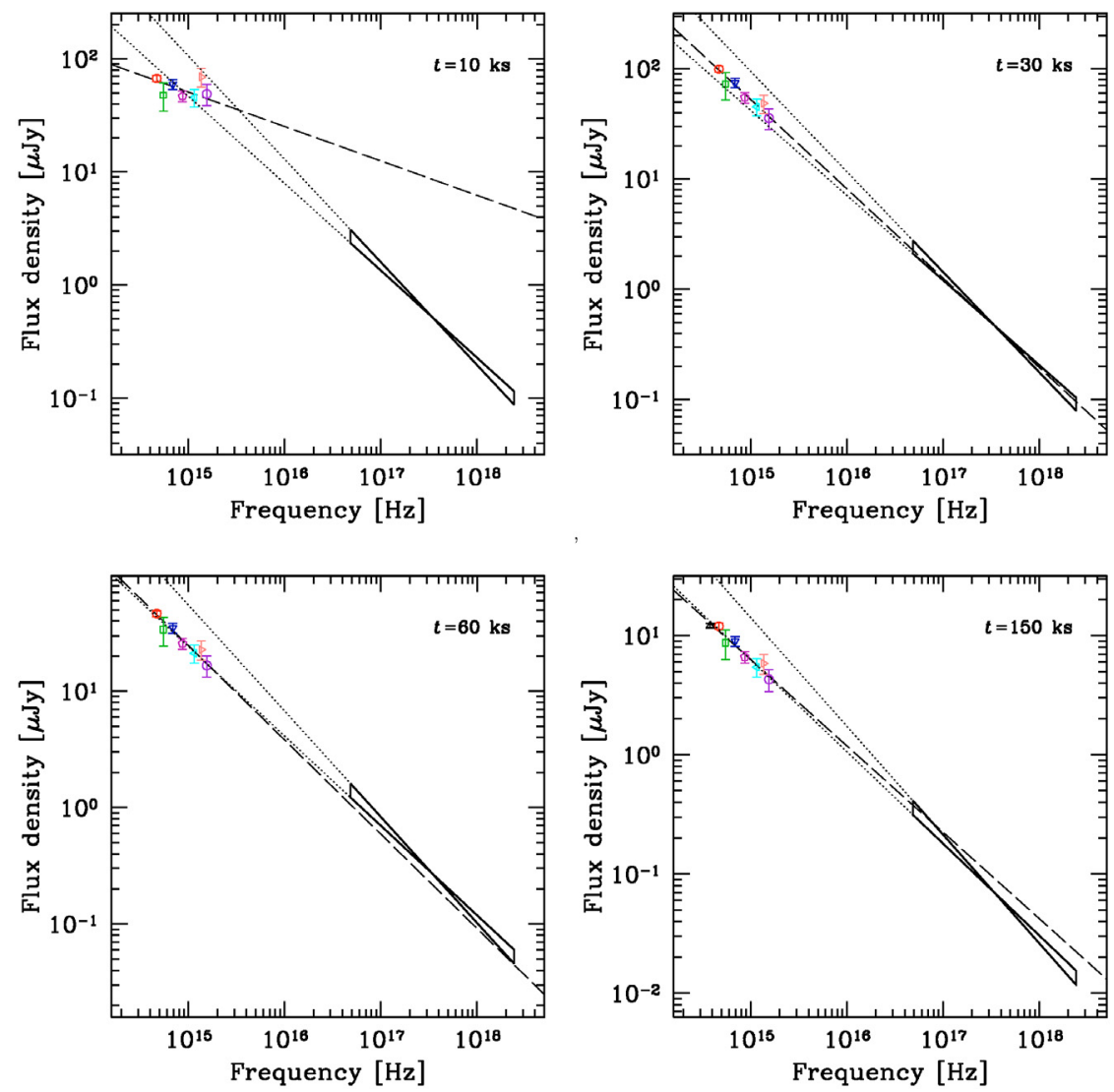

Fig. 7. Optical, ultraviolet and X-ray spectral energy distribution at 10,30, 60 and $150 \mathrm{ks}$ after the trigger. Optical and UV data, shown as points, have been corrected for the Galactic extinction along the line of sight $\left(A_{V}=0.07 \mathrm{mag}\right)$ and for a host extinction of $A_{V}=0.05 \mathrm{mag}$ with an SMC-like extinction curve. The SED at $150 \mathrm{ks}$ also contains the $I$ magnitude of the afterglow obtained by VLT observations (Della Valle et al. 2006). The solid lines define the cone corresponding to the $90 \%$ uncertainty on the spectral slope in the X-ray band. The extrapolation of the cone to the optical band is shown by the dotted lines. The dashed lines represent the best fit to the optical/UV data.

first break/peak, a time between the first and the second break and a time after the second break. The SED at $150 \mathrm{ks}$ also contains the I magnitude of the afterglow extrapolated from VLT observations (Della Valle et al. 2006). Infrared, optical and UV data have been corrected for the Galactic extinction along the line of sight $\left(A_{V}=0.07\right)$ and for a host extinction of $A_{V}=0.05 \pm 0.02$ with an SMC-like extinction curve. The value for the host galaxy extinction was computed in order to provide the best match for the last three SEDs. Results are shown in Fig. 7. The SED at $10 \mathrm{ks}$ implies a spectral break between the X-ray and optical bands. We note that since the early optical light curves are different at different frequencies the spectrum is changing, and $\beta_{\mathrm{opt}}$ gets redder with time for $t<T_{\mathrm{b}, 1}$. After $T_{\mathrm{b}, 1}$ all the SEDs are consistent with the optical and X-ray data belonging to the same power-law segment. Since the PC data do not show spectral evolution, the slope and opening of the cone representing the spectral distribution in the X-ray band and its uncertainty are constant and given by $\beta_{\mathrm{X}}=0.84 \pm 0.07$. The best fit of the optical and UV data has a slope of $\beta_{\mathrm{opt}}=0.30 \pm 0.14$ at $10 \mathrm{ks}$, and $\beta_{\text {opt }}=0.81 \pm 0.08$ at later times. So $\Delta \beta=\beta_{\mathrm{X}}-\beta_{\text {opt }}=0.54 \pm$ 0.16 at $10 \mathrm{ks}$, and $\Delta \beta=0.03 \pm 0.11$ at later times.

\section{Results and discussion}

\subsection{Prompt emission and early $X$-ray light curve}

The GRB 060614 prompt emission consists of an initial short hard emission structure with many bright peaks ( $\sim 5 \mathrm{~s}$ FWHM duration, $\left.E_{\mathrm{p}} \sim 300 \mathrm{keV}\right)$ followed by a longer $(\sim 170 \mathrm{~s})$, softer and highly variable bump which ends in a tail that smoothly matches the early and partly simultaneous X-ray light curve observed by XRT.

The XRT light curve shows strong spectral evolution during the very steep decay up to the end of the first orbit $(\sim 500 \mathrm{~s})$, then enters a nearly flat decay phase followed by steeper decay phases that will be discussed later in Sect. 4.3. We now focus only on data before $500 \mathrm{~s}$. The X-ray light curve over this time interval is not a power-law. In Table 6, we report fit results assuming an exponential shape, adopted for sake of simplicity only. The spectral fits suggest a physical interpretation of this steepening decline. The combined BAT/XRT spectrum is in fact well described by a Band model (or a cutoff power law) with decreasing peak energy $E_{\mathrm{p}}$. The passage of $E_{\mathrm{p}}$ through the XRT band would naturally produce a steepening in the light curve, since the flux density at $E>E_{\mathrm{p}}$ decreases faster than at $E<E_{\mathrm{p}}$. The width of the XRT bandpass $(0.2-10 \mathrm{keV})$ will make the transition smooth when considering the band-integrated flux. This behaviour is represented in Fig. 8, where we show the expected $0.2-10 \mathrm{keV}$ light curve for a Band spectrum with decreasing peak energy and normalization. We fixed the low and high-energy photon indices to $\alpha_{\text {Band }}=-1.1$ and $\beta_{\text {Band }}=-2.6$ (see third panel of Table 4). The time behaviour of $E_{\mathrm{p}}$ was obtained by interpolating the observed values (Fig. 8, inset), which show a regular decay $E_{\mathrm{p}} \propto t^{-\alpha}$ with $\alpha=2.04 \pm 0.11$. The agreement with the observed data is satisfactory, so that the light curve shape provides further support for the spectral evolution pattern 


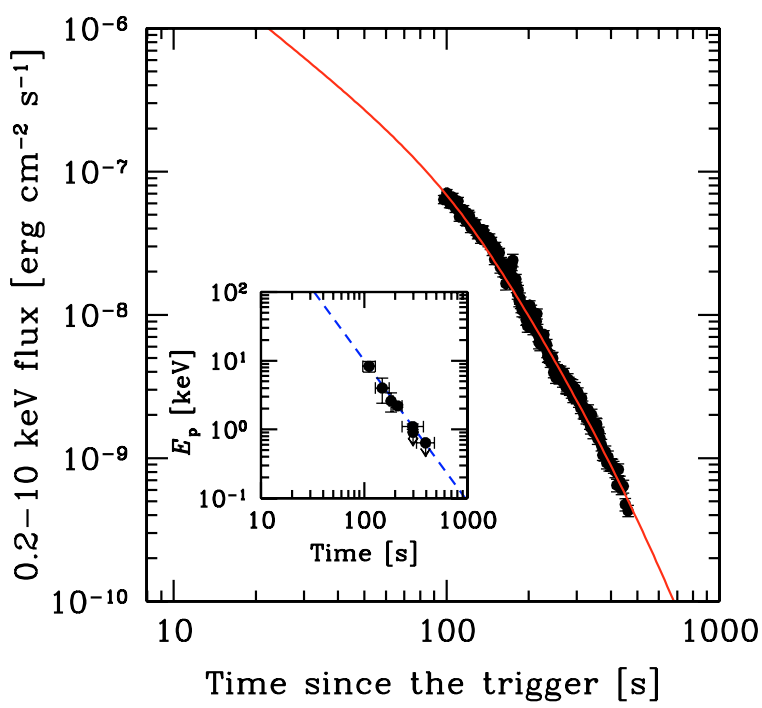

Fig. 8. Modeling of the early XRT light curve (black points) as the emission of a Band spectrum with evolving peak energy (red solid line). The $E_{\mathrm{p}}$ evolution law used is the best fit of the measured $E_{\mathrm{p}}$ values obtained through time resolved spectral analysis and presented in Table 4 . The $E_{\mathrm{p}}$ decay law with time is shown in the inset (blue dashed line).

described in Sect. 3.3. The observation of $E_{\mathrm{p}}$ passing through the XRT band during early XRT observations is indeed a natural possibility (Butler \& Kocevski 2007), being consistent with the well-known hard-to-soft evolution observed in the prompt emission spikes (Ford et al. 1995). Then, the measured $E_{\mathrm{p}}$ variation strongly supports the interpretation of the early XRT light curve of GRB 060614 as the low energy counterpart of the fading and softening tail seen by BAT. According to the analysis of Zhang et al. (2006b), the GRB 060614 tail behaviour cannot be explained as the simple superposition of high latitude radiation (Kumar \& Panaitescu 2000; Dermer 2004) and a possible prolonged and steady central engine emission (Zhang \& Mészáros 2004), but may be explained as the result of the cooling frequency decrease associated to adiabatic cooling of shock heated shells after an internal collision.

In their combined analysis of BAT and XRT data of Swift GRBs, O'Brien et al. (2006) and Willingale et al. (2006) concluded that all X-ray light curves can be well described by an exponential that relaxes into a power law, often with flares superimposed. There have also been a few cases with clearly detected exponential phases in the early XRT light curve (e.g. Vaughan et al. 2006). By analogy with GRB 060614, we suggest that also for these cases the light curve was shaped by the passage of the peak energy inside the XRT band. Hardness ratio plots can be used to test this hypothesis as done in the case of XRF 050416A by Mangano et al. (2007a) to show the consistency of Swift observations with a decaying $E_{\mathrm{p}}$ scenario.

The alternative time-dependent fit of early XRT data of GRB 060614 with the power law plus blackbody model (fourth panel in Table 4) may be unphysical, particularly given the fact that the broad band spectrum from 97 to $176 \mathrm{~s}$ is clearly fitted by a Band function, which may well continue up to $500 \mathrm{~s}$. The blackbody fit may be just a computational way to fit the curvature in a Band spectrum peaking within the narrow XRT energy range, and the observed decrease of the blackbody radius (related the normalization of the blackbody component) may be associated to the exit of the peak energy from the XRT energy band.
However, it is worth noticing that in GRB 060614 the long bump of the prompt emission peaks at about 40-50 s since the trigger. According to Kobayashi \& Zhang (2007), in the thick shell scenario the onset of the afterglow (and the corresponding peak in the light curve) would occur at about the fireball deceleration time $T_{\mathrm{dec}}=\left(3 E / 32 \pi \rho c^{5} \Gamma_{0}^{8}\right)^{1 / 3}$, where $E$ is the fireball total energy, $\rho$ is the mass density of the surrounding medium, $\Gamma_{0}$ is the initial Lorentz factor of the expanding fireball and $c$ is the speed of light. If we assume a radiation efficiency of shocks $\eta \sim 0.2$ we can estimate $E=E_{\text {iso }} / \eta$ with $E_{\text {iso }} \sim 2.5 \times 10^{51} \mathrm{erg}$ (see Sect. 4.2). Under the further assumptions $\rho \sim 10 m_{\mathrm{p}} \mathrm{cm}^{-3}$ (with $m_{\mathrm{p}}$ being the proton mass) we obtain $T_{\mathrm{dec}} \sim 45 \mathrm{~s}$ for $\Gamma_{0} \sim 100$.

On the other hand, the putative blackbody component observed after $100 \mathrm{~s}$ from the trigger has a temperature $k T_{\mathrm{bb}} \sim$ $0.9 \mathrm{keV}$ and radius $R_{\mathrm{bb}} \sim 2 \times 10^{11} \mathrm{~cm}$. This is similar to what expected from the slowly expanding $\left(\sim 10000 \mathrm{~km} \mathrm{~s}^{-1}\right) \mathrm{SN}$ shock front after break-out from the stellar surface if the $\mathrm{SN}$ radiation energy budget is of the order of $10^{51} \mathrm{erg}$. Then, the radiation we observe may be the superposition of standard internal shock activity up to $100 \mathrm{~s}$ from the trigger, afterglow onset and blackbody radiation from expanding $\mathrm{SN}$ shock. In this scenario, the absence of SN detection in the optical could be explained by a very under-luminous SN explosion due to fall back over the nuclear region of heavy elements (mainly $\mathrm{Ni}$ ) synthetized in the core collapse (Nomoto et al. 2004) or by a "dark hypernova" from a metal-poor massive star (Nomoto et al. 2007; Tominaga et al. 2007). Given the deep optical upper limits (Gal-Yam et al. 2006; Della Valle et al. 2006; Fynbo et al. 2006b), this would be one of the most under-luminous SN associated to a GRB ever detected, and one of the faintest $\mathrm{SNe}$ ever discovered.

\subsection{Energetics}

An accurate estimate of the isotropic equivalent energy radiated by GRBs requires the knowledge of their intrinsic average spectrum over the $1 \mathrm{keV}-10 \mathrm{MeV}$ energy band in the source rest frame. The narrow BAT sensitivity bandpass $(15-150 \mathrm{keV})$ makes it hard to detect GRB peak energies in most cases. The average spectrum of GRB 060614 is well fitted by a single powerlaw with photon index $\gtrsim 2$. This indicates that we are observing the high energy branch of the Band law generally used to describe GRBs spectra, and a $24 \mathrm{keV}$ upper limit to the average peak energy can be set in the observer frame (see Sect. 3.3). Moreover, we know that the burst presented strong spectral evolution and the peak energy decreased from $\sim 300 \mathrm{keV}$ measured during the initial group of peaks to $\sim 8 \mathrm{keV}$ during the BAT/XRT overlap. Then we expect that the average peak energy of the burst should be larger than $8 \mathrm{keV}$. With a peak energy in the 8-24 keV range, GRB 060614 is definitely an X-ray rich burst and may even be an X-ray flash (Lamb et al. 2005). A lower limit to the isotropic equivalent energy $E_{\text {iso }}$ can be obtained under the extreme assumption that the GRB emission below $24 \times$ $(1+z) \mathrm{keV}$ in the source rest frame is zero. An upper limit can be obtained under the assumption that the peak energy is below $1 \mathrm{keV}$ (i.e. extrapolating the $\Gamma \sim 2.13$ power law to low energies). In this way we obtain $1.8 \times 10^{51} \mathrm{erg}<E_{\text {iso }}<3.2 \times$ $10^{51} \mathrm{erg}$, and can reasonably assume $E_{\text {iso }}=(2.5 \pm 0.7) \times 10^{51} \mathrm{erg}$. According to this estimate and the limits on the average peak energy discussed above, GRB 060614 is consistent at about 2 sigma with the $E_{\mathrm{p}}^{\text {rest }}-E_{\text {iso }}$ correlation found by Amati et al. (2002) for long GRBs with known redshift and recently updated including a larger sample of events (Amati 2006; see also Amati et al. 2007). 
The rest-frame peak isotropic luminosity in the 30-10000 keV range (calculated assuming the broad band spectrum given by Konus-Wind, Golenetskii et al. 2006) is $L_{\mathrm{p}, \text { iso }}=5.3_{-1.8}^{+1.0} \times 10^{49} \mathrm{erg} \mathrm{s}^{-1}$. This value is consistent with the extrapolation to low luminosities of the $E_{\mathrm{p}}^{\text {rest }}-L_{\mathrm{p} \text {,iso }}$ relation found by Yonetoku et al. (2004).

The isotropic equivalent energy radiated during the initial hard episode of peaks $E_{\text {iso, } 1 \mathrm{p}}$ can also be estimated. The best fit photon index of the BAT spectrum of the initial group of peaks, $\Gamma \sim 1.6$, is in good agreement with the best fit photon index of Konus-Wind data over the same energy range and indicates that the peak energy was above the BAT energy range. Based on the $302 \mathrm{keV}$ peak energy detected by Konus-Wind, we obtain $E_{\text {iso, } 1 \mathrm{p}} \sim 3.5 \times 10^{50} \mathrm{erg}$. Then, only about one seventh of the isotropic energy was released during the initial group of peaks. As noted also by (Gehrels et al. 2006), the first hard episode of GRB 060614 is not consistent with the Amati correlation. This is not surprising, since the Amati correlation was computed using global, and not time-resolved, properties.

\subsection{Breaks and closure relations}

The XRT light curve for $t>4$ ks shows an initial flat decay with slope $\alpha_{\mathrm{A}}=0.11 \pm 0.05$ and a constant spectral energy index $\beta_{\mathrm{A}}=0.84 \pm 0.10$, a steepening to a slope $\alpha_{\mathrm{B}}=1.03 \pm 0.02$ at $36.6 \pm 2.4 \mathrm{ks}$ without significant spectral evolution $\left(\beta_{\mathrm{B}}=0.96 \pm\right.$ 0.16 ) and a final steepening to a slope $\alpha_{\mathrm{C}}=2.13 \pm 0.07$ at $104 \pm 22 \mathrm{ks}$ without significant changes in the spectrum $\left(\beta_{\mathrm{C}}=\right.$ $0.77 \pm 0.12)$. This behaviour resembles the typical behaviour of Swift detected X-ray afterglows (Nousek et al. 2006; O'Brien et al. 2006). The slow decay rate of phase A can be interpreted as the effect of forward shock refreshing mechanisms, likely ending at the time of the first break (Zhang et al. 2006a; Nousek et al. 2006; Panaitescu et al. 2006). The slope of the phase B decay is intermediate between the value expected in the cases $v_{\mathrm{X}}<v_{\mathrm{c}}$ and $v_{\mathrm{X}}>v_{\mathrm{c}}\left(v_{\mathrm{c}}\right.$ being the synchrotron cooling frequency), namely $\alpha=3 \beta / 2=1.26 \pm 0.10$ and $\alpha=3 \beta / 2-1 / 2=0.76 \pm$ 0.10 . Since phase B lasted for a relatively short time (only half a decade), the value of $\alpha_{\mathrm{B}}$ may be affected by systematic errors due to the choice of the fitting function (we chose for simplicity a piecewise-connected power law, while the real shape of the transition will be smooth; Granot \& Sari 2002). The hard spectral shape suggests that $v_{\mathrm{c}}$ is above the X-ray band.

The striking feature of the multi-band light curve, however, is the presence of an achromatic break at $T_{\mathrm{b}, 2}$, observed simultaneously in the XRT light curve as well as in the UVOT and $R$-band ones. An achromatic transition is expected when the edge of the jet enters the visible portion of the emitting surface. Note that Della Valle et al. (2006) found a break in the optical afterglow light curve at $119 \pm 3.4 \mathrm{ks}$ with pre-break slope $1.08 \pm 0.03$ and post-break slope $2.48 \pm 0.07$. Then, our simultaneous fit of the UVOT light curves with the $R$-band light curve from Della Valle et al. (2006) points toward the consistency of all the optical/UV data with a break at $117.2 \pm$ $4.4 \mathrm{ks}$. This confirms the jet-break nature of the second break of our X-ray light curve. The X-ray and optical slopes after the break are roughly consistent with each other, and consistent with the decay predicted after a jet break. To our knowledge, this is one of the best examples of a jet break for a Swift burst. A jet-break at $\sim 104 \mathrm{ks}$ implies a jet opening angle of $\vartheta_{\text {jet }}=$ $0.161\left[t_{\mathrm{b}} /(1+z)\right]^{3 / 8}\left(n \eta_{\gamma} / E_{52}\right)^{1 / 8} \mathrm{rad}$ (Sari et al. 1999) where, $t_{\mathrm{b}}$ is the break time in days, $E_{52}$ is the isotropic energy release in units of $10^{52} \mathrm{erg}, n$ is the particle density of the circumburst matter in $\mathrm{cm}^{-3}$ and $\eta_{\gamma}$ is the conversion efficiency of internal energy to gamma-rays. For an isotropic energy $E_{\text {iso }} \sim 2.5 \times$ $10^{51}$ erg we obtain $\vartheta_{\text {jet }}=10.5^{\circ}(n / 3)^{1 / 8}\left(\eta_{\gamma} / 0.2\right)^{1 / 8}$. This corresponds to a beaming-corrected energy $E_{\gamma}=E_{\text {iso }}\left(1-\cos \vartheta_{\text {jet }}\right) \sim$ $4.2 \times 10^{49}$ erg and makes GRB 060614 roughly consistent with the $E_{\mathrm{p}}^{\text {rest }}-E_{\gamma}$ relation originally found by Ghirlanda et al. (2004) and re-investigated by Liang \& Zhang (2005) and Ghirlanda et al. (2007). Note that the beaming-corrected energy of GRB 060614 is lower than typical $E_{\gamma}$ of long GRBs $\left(\sim 10^{51} \mathrm{erg}\right)$ and comparable to the beaming-corrected energy of the short burst GRB 051221A (Burrows et al. 2006).

The interpretation of the first break of the X-ray light curve as a hydrodynamical break due to the end of forward shock refreshing mechanisms would also require an achromatic break. Indeed, the optical and ultraviolet light curves of the afterglow show another common break at about $30 \mathrm{ks}$, nearly simultaneous with the first X-ray break (that occurs at about $37 \mathrm{ks}$ ). However, the initial slope $\alpha_{\mathrm{A}}$ is dependent upon wavelength at optical frequencies. This implies that the spectrum was changing at these wavelengths around $t \sim 10 \mathrm{ks}$. In particular, since the UV light curves are initially decaying, while at lower frequencies a clear rise is observed, the spectrum is evolving from blue to red. A way to explain this behaviour is the passage of a break frequency through the optical/UV band. This kind of transition should also be accompanied by a light curve slope change as well, but is not achromatic. The observed behaviour of all our afterglow light curves could be accounted for if the break frequency passed through the observed band slightly earlier than the hydrodynamical break. In this scenario the observed initial break/peak in the UVOT and VLT light curves would be due to the superposition of the two effects (causing the change in the spectrum) while the initial break in the X-ray light curve would be purely hydrodynamical (with no spectral evolution across it, as observed). Which kind of break frequency can produce the observed behaviour? The simplest possibility is that we were observing the injection frequency $v_{i}$, that is the frequency at which the lowest-energy electrons are radiating. For $v<v_{\mathrm{i}}$, where the spectrum is very blue $\left(F_{v} \propto v^{+1 / 3}\right)$, rising light curves are expected, while a standard decay occurs for $v>v_{\mathrm{i}}$. As the injection frequency moves to longer wavelengths, the curves above $v_{\mathrm{i}}$ would be decaying, while the curves below $v_{\mathrm{i}}$ would be still rising. The very blue spectrum is confirmed by our spectral analysis of the early-time SED (which has $\beta_{\text {opt }}=0.30 \pm 0.14$ ). If this interpretation is correct, this could be the first time for which the injection frequency was directly observed in the optical band.

The other possibility is that the change in the spectral shape around $t=10 \mathrm{ks}$ is due to the passage of the cooling frequency. For this model to work, however, the shocked electron power law slope should be $p=2 \beta_{\mathrm{X}} \sim 1.7$. Using the formulae of Dai $\&$ Cheng (2001) and Zhang \& Mészáros (2004) to predict the light curve slope in case of forward shock propagation in the slow cooling regime in a uniform interstellar medium when $p<2$, we obtain $\alpha_{B}$ between 0.9 and 1 , roughly consistent with the observation. The main problem of this interpretation is that the required $p$ value (1.7) is substantially lower than the value consistent with the jet break interpretation of the second break (2.2). A temporal evolution of the shocked electron power law slope within the first $30 \mathrm{ks}$ could reconcile the models. A $p$ change, i.e. a shock microphysics change, during an afterglow evolution is not an implausible event and could be related to changes in the dynamics of the shock like those associated with the end of shock refreshing mechanisms. This would however produce a change in the X-ray spectrum as well, which is not observed. 


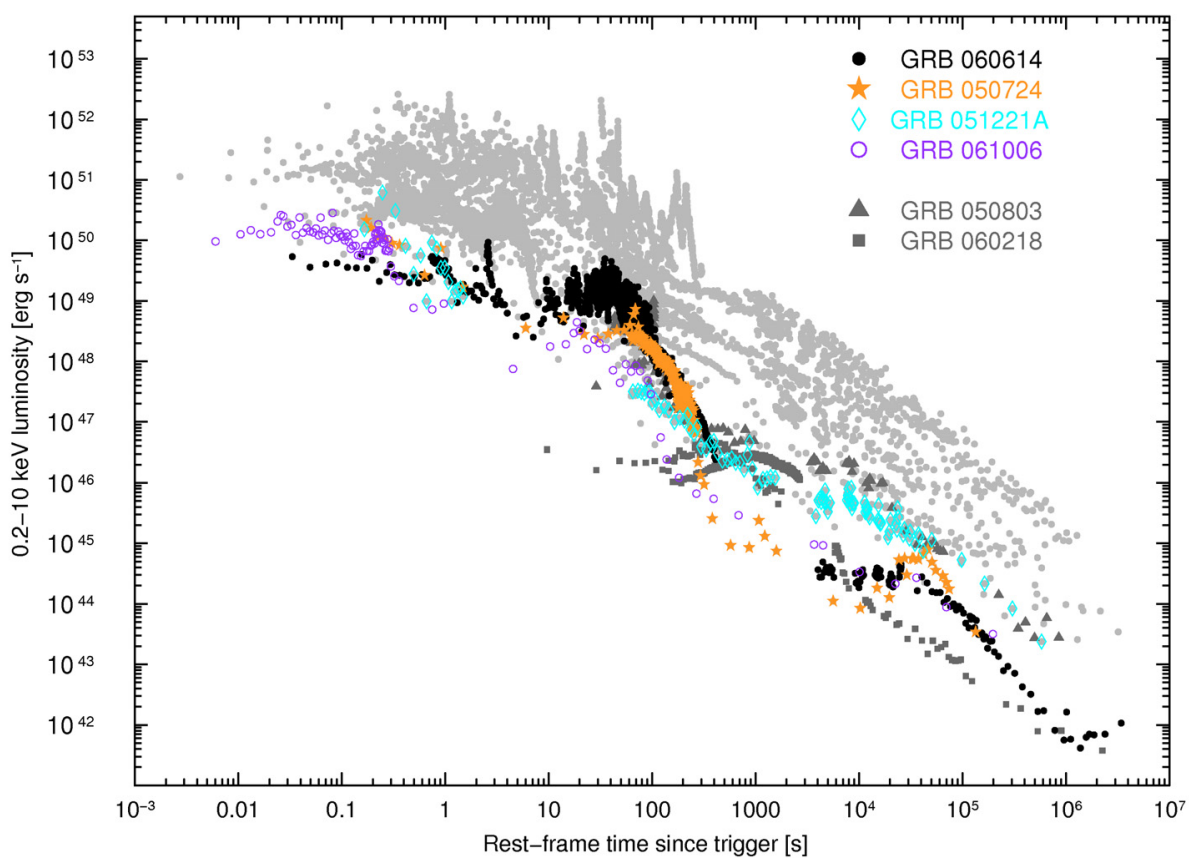

Fig. 9. Rest-frame luminosity light curves of several GRBs detected by Swift: GRB 060614 (black points), the 3 short-duration GRBs with secure redshift (GRB 050724, GRB 051221A, and GRB 061006) and 37 long bursts with known redshift (light and dark grey curves). The only two long GRBs with redshift $z<0.5$ are evidenced (dark grey curves). Note that the sample contains other 5 long bursts with $0.5<z<1$. Each light curve consists of $i$ ) the BAT light curve of the prompt emission extracted in the $15-150 \mathrm{keV}$ energy band, extrapolated to flux in the $0.2-10 \mathrm{keV}$ energy band using the best fit parameters of the BAT spectrum and then converted into $0.2-10 \mathrm{keV}$ luminosity using the appropriate $k$-correction; $i i)$ the XRT light curves, converted to $0.2-10 \mathrm{keV} \mathrm{lu}-$ minosity with the appropriate $k$-correction required for the best fit model of the XRT spectra. See Mangano et al. (2007b) for further details.

\subsection{Comparison with other bursts}

Despite the contrasting properties of GRB 060614, which make it difficult to classify unambiguously, its afterglow does not show anomalous features. Phenomenologically, its X-ray light curve shows all the "canonical" components identified in long GRB afterglows (Nousek et al. 2006; O'Brien et al. 2006; Willingale et al. 2006). Furthermore, its properties are in remarkable agreement with the predictions of jetted fireball models (Sari et al. 1998, 1999). However, several recent studies have outlined that the X-ray afterglows of short GRBs show the same basic features as well: power law decay with changing slopes, X-ray flares (GRB 050724: Campana et al. 2006b; Grupe et al. 2006; GRB 051210: La Parola et al. 2006), shallow decay phases (GRB 060313: Roming et al. 2006; GRB 061006: Schady et al. 2006), breaks (GRB 061201: Marshall et al. 2006; GRB 061006: Schady et al. 2006) and possibly jet breaks (GRB 051221A: Burrows et al. 2006). This makes it difficult to infer conclusions about the nature of GRB 060614 based on its light curve properties. An interesting feature observed in a fraction of shortduration GRBs is the existence of a soft hump following the initial short, hard spike (Norris \& Bonnell 2006). As noted by several authors (Gehrels et al. 2006; Zhang et al. 2007), this feature resembles what observed in GRB 060614, which may be an extreme case of such behaviour.

In Fig. 9 we plotted the (rest-frame) luminosity light curves of several GRBs detected by Swift, including GRB 060614, the 3 short GRBs with secure spectroscopic redshift ${ }^{3}$ (GRB 050724, GRB 051221A, GRB 061006), and 37 long GRBs with measured redshift (mostly from Mangano et al. 2007b).

The light curve of GRB 060614 is strikingly similar to those of GRB 050724 and GRB 061006, in terms of both shape and luminosity. Note that these two bursts showed an extended soft emission episode (Barthelmy et al. 2005c; Campana et al. 2006b; Schady et al. 2006). We note, however, that the shape of the GRB 060614 light curve is quite similar to that of several longduration GRBs. In terms of luminosity, taken at a face value,

3 We require the existence of an optical counterpart in order to have a certain identification of the host galaxy. it looks that GRB 060614 is at the faint end of the distribution, being $~ 50$ times fainter than the average. This comparison, however, is a bit misleading given the big difference in the (average) redshift of most long-duration bursts and GRB 060614. Among the plotted objects, only GRB 060218 and GRB 050803 have $z<0.5(z=0.0331$ and $z=0.422$, respectively; Mirabal et al. 2006; Bloom et al. 2005), and, not surprisingly, they have both faint light curves, comparable in luminosity to GRB 060614 and to the short GRBs. Of course, given the steep GRB luminosity function (Firmani et al. 2004), closeby objects are most likely to be faint on the average, given the small sampled volume at low redshift. It is also whortwhile to mention that one of the striking features of GRB 060614, namely the lack of a bright associated supernova, would pass unnoticed at larger redshift, so that the number of SN-less event is actually poorly constrained. Only the detection of a statistically significant number of longduration events at moderate/low redshift will make it possible a meaningful comparison.

\section{Conclusions}

GRB 060614 is a very peculiar, nearby $(z=0.125)$ burst. Despite the long duration of its prompt emission $\left(T_{90}=102 \pm 5 \mathrm{~s}\right)$, it presents null time lags in the BAT bandpass (similar to short bursts) and has no evidence of an associated supernova down to very deep limits $\left(M_{V} \sim-14\right)$. GRB 060614 lies close to the region occupied by short bursts in the time-lags/peak-luminosity plane, but, on the other hand, satisfies the $E_{\text {iso }}-E_{\mathrm{p}}^{\text {rest }}$ (Amati) correlation, that holds for long bursts only. Its BAT light curve shows a prolonged softening tail that smoothly matches the partly simultaneous early XRT light curve. The temporal decay of this tail is well described by an exponential function, with an $e$-folding time of $\sim 76 \mathrm{~s}$, and the spectral evolution is associated with the drift of the peak energy of the Band function towards lower energy and crossing the XRT energy band.

The X-ray, UV and optical afterglow light curves and SEDs of GRB 060614 are well interpreted within the standard afterglow theory. In particular, the afterglow of GRB 060614 shows a truly achromatic break from optical to X-ray frequencies at $\sim 104$ ks after the trigger, fully consistent with being a jet break. 
The burst satisfies the $E_{\gamma}-E_{\mathrm{p}}^{\mathrm{rest}}$ (Ghirlanda) correlation. The observed softening of the optical/UV afterglow before $30 \mathrm{ks}$ can be interpreted as an evidence of passage of the injection frequency through the optical band.

The good agreement of the afterglow of GRB 060614 with the jetted fireball models does not allow us to draw firm conclusions on the nature of the event and its possible progenitor, but is indeed remarkable since many Swift bursts hardly reconcile with the very same models.

Acknowledgements. This work is supported at INAF by funding from ASI on grant number I/R/039/04 and from MIUR grant 2005025417, at Penn State by NASA contract NASS5-00136, and at the University of Leicester by the Particle Physics and Astronomy Research Council. We gratefully acknowledge the contribution of dozens of members of the XRT team at OAB, PSU, UL, GSFC, ASDC and our sub-contractors, who helped make this instrument possible. D.M. acknowledges support from the Instrument Center for Danish Astrophysics.

\section{References}

Amati, L. 2006, MNRAS, 372, 233

Amati, L., Frontera, F., Tavani, M., et al. 2002, A\&A, 390, 81

Amati, L., Della Valle, M., Frontera, F., et al. 2007, A\&A, 463, 913

Band, D., Matteson, J., Ford, L., et al. 1993, ApJ, 413, 281

Barthelmy, S., Gehrels, N., Norris, J., \& Sakamoto, T. 2005a, GRB Coordinates Network, 4401, 1

Barthelmy, S. D., Barbier, L. M., Cummings, J. R., et al. 2005b, Space Sci. Rev., 120,143

Barthelmy, S. D., Chincarini, G., Burrows, D. N., et al. 2005c, Nature, 438, 994

Barthelmy, S., Barbier, L., Cummings, J., et al. 2006, GRB Coordinates Network, 5256, 1

Bloom, J. S., Perley, D., Foley, R., et al. 2005, GRB Coordinates Network, 3758, 1

Brown, P. J. 2006, GRB Coordinates Network, 5286, 1

Burrows, D. N., Hill, J. E., Nousek, J. A., et al. 2005, Space Sci. Rev., 120, 165

Burrows, D. N., Grupe, D., Capalbi, M., et al. 2006, ApJ, 653, 468

Butler, N. R., \& Kocevski, D. 2007, ApJ, in press [arXiv: astro-ph/0612564]

Campana, S., Mangano, V., Blustin, A. J., et al. 2006a, Nature, 442, 1008

Campana, S., Tagliaferri, G., Lazzati, D., et al. 2006b, A\&A, 454, 113

Christensen, L., Hjorth, J., \& Gorosabel, J. 2004, A\&A, 425, 913

Cobb, B. E. 2006, GRB Coordinates Network, 5259, 1

Cobb, B. E., \& Bailyn, C. D. 2006, GRB Coordinates Network, 5282, 1

Cobb, B. E., Bailyn, C. D., van Dokkum, P. G., \& Natarajan, P. 2006, ApJ, 651, L85

Dai, Z. G., \& Cheng, K. S. 2001, ApJ, 558, L109

Della Valle, M., Chincarini, G., Panagia, N., et al. 2006, Nature, 444, 1050

Dermer, C. D. 2004, ApJ, 614, 284

Dickey, J. M., \& Lockman, F. J. 1990, ARA\&A, 28, 215

Donaghy, T. Q., Lamb, D. Q., Sakamoto, T., et al. 2006, ApJ, submitted [arXiv: astro-ph/0605570]

Firmani, C., Avila-Reese, V., Ghisellini, G., \& Tutukov, A. V. 2004, ApJ, 611, 1033

Ford, L. A., Band, D. L., Matteson, J. L., et al. 1995, ApJ, 439, 307

French, J., Melady, G., Hanlon, L., Jelínek, M., \& Kubánek, P. 2006, GRB Coordinates Network, 5257, 1

Fugazza, D., Malesani, D., Romano, P., et al. 2006, GRB Coordinates Network, 5276,1

Fynbo, J. P. U., Thöne, C. C., Jensen, B. L., et al. 2006a, GRB Coordinates Network, 5277, 1

Fynbo, J. P. U., Watson, D., Thöne, C. C., et al. 2006b, Nature, 444, 1047

Gal-Yam, A., Fox, D. B., Price, P. A., et al. 2006, Nature, 444, 1053

Galama, T. J., Vreeswijk, P. M., van Paradijs, J., et al. 1998, Nature, 395, 670

Gehrels, N., Chincarini, G., Giommi, P., et al. 2004, ApJ, 611, 1005

Gehrels, N., Norris, J. P., Barthelmy, S. D., et al. 2006, Nature, 444, 1044

Ghirlanda, G., Ghisellini, G., \& Lazzati, D. 2004, ApJ, 616, 331

Ghirlanda, G., Nava, L., Ghisellini, G., \& Firmani, C. 2007, A\&A, 466, 127

Golenetskii, S., Aptekar, R., Mazets, E., et al. 2006, GRB Coordinates Network, 5264,1

Granot, J., \& Sari, R. 2002, ApJ, 568, 820

Grimm, H.-J., Gilfanov, M., \& Sunyaev, R. 2003, MNRAS, 339, 793

Grupe, D., Burrows, D. N., Patel, S. K., et al. 2006, ApJ, 653, 462
Grupe, D., Gronwall, C., Wang, X.-Y., et al. 2007, ApJ, in press [arXiv: astro-ph/0611240]

Hill, J. E., Burrows, D. N., Nousek, J. A., et al. 2004, in X-Ray and GammaRay Instrumentation for Astronomy XIII, Proceedings of the SPIE, ed. K. A. Flanagan, \& O. H. W. Siegmund, 5165, 217

Hjorth, J., Sollerman, J., Møller, P., et al. 2003, Nature, 423, 847

Holland, S. T. 2006a, GRB Coordinates Network, 5281, 1

Holland, S. T. 2006b, GRB Coordinates Network, 5255, 1

King, A., Olsson, E., \& Davies, M. B. 2007, MNRAS, 374, L34

Kobayashi, S., \& Zhang, B. 2007, ApJ, 655, 973

Krimm, H., Parsons, A., \& Markwardt, C. 2004, Swift-BAT Ground Analysis Software Manual

Krimm, H., Barbier, L., Barthelmy, S., et al. 2006, GRB Coordinates Network, 5704, 1

Kumar, P., \& Panaitescu, A. 2000, ApJ, 541, L51

La Parola, V., Mangano, V., Fox, D., et al. 2006, A\&A, 454, 753

Lamb, D. Q., Donaghy, T. Q., \& Graziani, C. 2005, ApJ, 620, 355

Lazzati, D., Ramirez-Ruiz, E., \& Ghisellini, G. 2001, A\&A, 379, L39

Lee, W. H., Ramirez-Ruiz, E., \& Page, D. 2004, ApJ, 608, L5

Liang, E., \& Zhang, B. 2005, ApJ, 633, 611

Malesani, D., Tagliaferri, G., Chincarini, G., et al. 2004, ApJ, 609, L5

Malesani, D., Covino, S., Campana, S., et al. 2006, GRB Coordinates Network, 5261, 1

Mangano, V., La Parola, V., Troja, E., et al. 2006, GRB Coordinates Network, 5254,1

Mangano, V., La Parola, V., Cusumano, G., et al. 2007a, ApJ, 654, 403

Mangano, V., La Parola, V., Troja, E., et al. 2007b, Nuovo Cimento, in press [arXiv:astro-ph/0701812]

Marshall, F., Perri, M., Stratta, G., et al. 2006, GCNR, 18, 1

Mirabal, N., Halpern, J. P., An, D., Thorstensen, J. R., \& Terndrup, D. M. 2006, ApJ, 643, L99

Nomoto, K., Maeda, K., Mazzali, P. A., et al. 2004, in Stellar Collapse, ed. C. L. Fryer, Astrophysics and Space Science Library (Dordrecht: Kluwer Academic), 302, 277

Nomoto, K., Tominaga, N., Tanaka, M., et al. 2007, Nuovo Cimento, in press [arXiv: astro-ph/0702472]

Norris, J. P. 2002, ApJ, 579, 386

Norris, J. P., \& Bonnell, J. T. 2006, ApJ, 643, 266

Nousek, J. A., Kouveliotou, C., Grupe, D., et al. 2006, ApJ, 642, 389

O'Brien, P. T., Willingale, R., Osborne, J., et al. 2006, ApJ, 647, 1213

Palmer, D., Barbier, L., Barthelmy, S. D., et al. 2006, GRB Coordinates Network, 5905, 1

Panaitescu, A., Mészáros, P., Gehrels, N., Burrows, D., \& Nousek, J. 2006, MNRAS, 366, 1357

Parsons, A. M., Cummings, J. R., Gehrels, N., et al. 2006, GRB Coordinates Network, 5252, 1

Price, P. A., Berger, E., \& Fox, D. B. 2006, GRB Coordinates Network, 5275, 1

Romano, P., Campana, S., Chincarini, G., et al. 2006, A\&A, 456, 917

Roming, P. W. A., Kennedy, T. E., Mason, K. O., et al. 2005, Space Sci. Rev., 120,95

Roming, P. W. A., Vanden Berk, D., Pal'shin, V., et al. 2006, ApJ, 651, 985

Rosswog, S., Ramirez-Ruiz, E., \& Davies, M. B. 2003, MNRAS, 345, 1077

Sari, R., Piran, T., \& Narayan, R. 1998, ApJ, 497, L17

Sari, R., Piran, T., \& Halpern, J. P. 1999, ApJ, 519, L17

Schady, P., Breeveld, A., Poole, T. S., et al. 2006, GCNR, 6, 1

Schlegel, D. J., Finkbeiner, D. P., \& Davis, M. 1998, ApJ, 500, 525

Schmidt, B., Peterson, B., \& Lewis, K. 2006, GRB Coordinates Network, 5258, 1

Sollerman, J., Östlin, G., Fynbo, J. P. U., et al. 2005, New Astron., 11, 103

Stanek, K. Z., Matheson, T., Garnavich, P. M., et al. 2003, ApJ, 591, L17

Thoene, C. C., Fynbo, J. P. U., Jensen, B. L., et al. 2006, GRB Coordinates Network, 5272, 1

Tominaga, N., Maeda, K., Umeda, H., et al. 2007, ApJ, 657, L77

Vaughan, S., Goad, M. R., Beardmore, A. P., et al. 2006, ApJ, 638, 920

Villasenor, J. S., Lamb, D. Q., Ricker, G. R., et al. 2005, Nature, 437, 855

Willingale, R., O’Brien, P. T., Osborne, J. P., et al. 2006, ApJ, submitted [arXiv: astro-ph/0612031]

Yonetoku, D., Murakami, T., Nakamura, T., et al. 2004, ApJ, 609, 935

Zhang, B., \& Mészáros, P. 2004, Int. J. Mod. Phys. A, 19, 2385

Zhang, B., Fan, Y. Z., Dyks, J., et al. 2006a, ApJ, 642, 354

Zhang, B.-B., Liang, E.-W., \& Zhang, B. 2006b, ApJ, submitted [arXiv:astro-ph/0612246]

Zhang, B., Zhang, B.-B., Liang, E.-W., et al. 2007, ApJ, 655, L25 


\section{Online Material}


V. Mangano et al.: Swift observations of GRB 060614, Online Material p 2

Table 1. BAT and XRT data log.

\begin{tabular}{llllrr}
\hline \hline Sequence & Obs/Mode & Start time (UT) & End time (UT) & Exposure (s) $^{\text {Start time }}{ }^{a}$ \\
\hline 00214805000 & BAT & $2006-06-1412: 39: 49$ & 2006-06-14 12:51:51 & 759 & -240 \\
\hline 00214805000 & XRT/WT & $2006-06-1412: 45: 25$ & $2006-06-1420: 23: 16$ & 414 & 97 \\
00214805000 & XRT/PC & $2006-06-1413: 57: 39$ & $2006-06-1420: 45: 06$ & 10628 & 4449 \\
00214805001 & XRT/PC & $2006-06-1500: 05: 06$ & $2006-06-1523: 59: 57$ & 17642 & 40878 \\
00214805002 & XRT/PC & $2006-06-1600: 08: 04$ & $2006-06-1722: 49: 57$ & 42687 & 127456 \\
00214805003 & XRT/PC & $2006-06-1800: 05: 06$ & $2006-06-2023: 06: 58$ & 57900 & 300078 \\
00214805004 & XRT/PC & $2006-06-2100: 30: 32$ & $2006-06-2123: 13: 57$ & 16781 & 560804 \\
00214805005 & XRT/PC & $2006-06-2200: 27: 31$ & $2006-06-2223: 25: 58$ & 20112 & 647023 \\
$00214805006-08$ & XRT/PC & $2006-06-2302: 10: 33$ & $2006-06-2523: 34: 58$ & 37183 & 739604 \\
$00214805009-10$ & XRT/PC & $2006-06-2600: 44: 32$ & $2006-06-2723: 34: 56$ & 28171 & 993644 \\
00214805011 & XRT/PC & $2006-06-2712: 21: 21$ & $2006-06-2723: 52: 56$ & 7356 & 1121852 \\
$00214805012-17$ & XRT/PC & $2006-06-2801: 03: 23$ & $2006-06-3022: 37: 58$ & 61535 & 1167575 \\
$00214805018-19$ & XRT/PC & $2006-07-0101: 35: 19$ & $2006-07-0322: 54: 56$ & 65247 & 1428691 \\
$00214805020-21$ & XRT/PC & $2006-07-0400: 12: 31$ & $2006-07-0523: 06: 57$ & 40088 & 1682922 \\
$00214805022-23$ & XRT/PC & $2006-07-0600: 27: 57$ & $2006-07-0623: 11: 57$ & 18101 & 1856649 \\
$00214805024-28$ & XRT/PC & $2006-07-0700: 31: 50$ & $2006-07-1018: 33: 58$ & 32353 & 1943282 \\
$00214805029-30$ & XRT/PC & $2006-07-1405: 38: 50$ & $2006-07-1722: 32: 56$ & 18815 & 2566501 \\
$00214805031-37$ & XRT/PC & $2006-07-1904: 51: 31$ & $2006-08-0721: 37: 56$ & 38602 & 2995662 \\
\hline
\end{tabular}

${ }^{a}$ Seconds since the trigger. 
Table 2. UVOT data.

\begin{tabular}{|c|c|c|c|c|c|c|c|}
\hline Sequence & Obs/Filter & Start time (UT) & End time (UT) & Exposure (s) & Start time $^{a}$ & $\mathrm{mag}^{b}$ & err \\
\hline 00214805000 & UVOT/White & $2006-06-14$ 12:45:33 & 2006-06-14 12:47:11 & 97 & 104 & 18.41 & 0.11 \\
\hline 00214805000 & UVOT/V & 2006-06-14 12:47:15 & 2006-06-14 12:50:30 & 191 & 206.5 & $>19.70$ & - \\
\hline 00214805000 & UVOT/UVM2 & 2006-06-14 13:57:40 & 2006-06-14 14:00:60 & 22 & 4431 & $>17.70$ & - \\
\hline 00214805000 & UVOT/UVW1 & 2006-06-14 14:01:05 & 2006-06-14 14:04:25 & 197 & 4636 & 18.49 & 0.31 \\
\hline 00214805000 & UVOT/U & 2006-06-14 14:04:29 & 2006-06-14 14:07:49 & 197 & 4840 & 18.79 & 0.16 \\
\hline 00214805000 & UVOT/B & 2006-06-14 14:07:54 & 2006-06-14 14:11:14 & 197 & 5045 & 19.82 & 0.21 \\
\hline 00214805000 & UVOT/White & 2006-06-14 14:11:18 & 2006-06-14 14:14:38 & 197 & 5249 & 18.25 & 0.06 \\
\hline 00214805000 & UVOT/UVW2 & $2006-06-14$ 14:14:43 & $2006-06-14$ 14:18:03 & 197 & 5454 & 18.26 & 0.23 \\
\hline 00214805000 & UVOT/V & 2006-06-14 14:18:08 & $2006-06-14$ 14:21:28 & 197 & 5659 & 19.34 & 0.27 \\
\hline 00214805000 & UVOT/UVM2 & 2006-06-14 14:21:32 & 2006-06-14 14:24:52 & 197 & 5863 & 17.78 & 0.18 \\
\hline 00214805000 & UVOT/UVW1 & 2006-06-14 14:24:57 & 2006-06-14 14:28:17 & 197 & 6068 & 18.32 & 0.25 \\
\hline 00214805000 & UVOT/U & 2006-06-14 14:28:22 & 2006-06-14 14:30:57 & 153 & 6272.5 & 18.55 & 0.19 \\
\hline 00214805000 & UVOT/B & 2006-06-14 15:34:07 & 2006-06-14 15:39:07 & 295 & 10218 & 19.46 & 0.13 \\
\hline 00214805000 & UVOT/B & 2006-06-14 15:39:11 & 2006-06-14 15:44:11 & 295 & 10522 & 19.51 & 0.13 \\
\hline 00214805000 & UVOT/B & 2006-06-14 15:44:13 & 2006-06-14 15:49:13 & 295 & 10824 & 19.56 & 0.12 \\
\hline 00214805000 & UVOT/White & 2006-06-14 15:49:18 & 2006-06-14 15:54:18 & 293 & 11129 & 18.34 & 0.07 \\
\hline 00214805000 & UVOT/White & 2006-06-14 15:54:21 & 2006-06-14 15:59:21 & 295 & 11432 & 18.36 & 0.05 \\
\hline 00214805000 & UVOT/White & 2006-06-14 15:59:25 & 2006-06-14 16:04:25 & 295 & 11736 & 18.24 & 0.08 \\
\hline 00214805000 & UVOT/UVW2 & 2006-06-14 16:04:30 & 2006-06-14 16:13:26 & 528 & 12041 & 18.10 & 0.48 \\
\hline 00214805000 & UVOT/V & 2006-06-14 17:10:30 & 2006-06-14 17:15:30 & 295 & 16001 & 19.46 & 0.25 \\
\hline 00214805000 & UVOT/V & 2006-06-14 17:15:34 & 2006-06-14 17:20:34 & 295 & 16305 & 19.21 & 0.21 \\
\hline 00214805000 & UVOT/V & 2006-06-14 17:20:37 & 2006-06-14 17:25:37 & 295 & 16608 & 19.32 & 0.22 \\
\hline 00214805000 & UVOT/UVM2 & $2006-06-14$ 17:25:41 & 2006-06-14 17:40:41 & 886 & 16912 & 18.62 & 0.81 \\
\hline 00214805000 & UVOT/UVW1 & 2006-06-14 17:40:48 & 2006-06-14 17:52:21 & 682 & 17819.5 & 18.44 & 0.27 \\
\hline 00214805000 & UVOT/U & 2006-06-14 18:46:57 & 2006-06-14 18:51:57 & 295 & 21788 & 18.56 & 0.12 \\
\hline 00214805000 & UVOT/U & 2006-06-14 18:51:60 & 2006-06-14 18:56:60 & 295 & 22091 & 18.48 & 0.12 \\
\hline 00214805000 & UVOT/U & 2006-06-14 18:57:03 & 2006-06-14 19:02:03 & 295 & 22394 & 18.53 & 0.11 \\
\hline 00214805000 & UVOT/B & 2006-06-14 19:02:09 & 2006-06-14 19:07:09 & 295 & 22700 & 19.54 & 0.14 \\
\hline 00214805000 & UVOT/B & 2006-06-14 19:07:11 & 2006-06-14 19:12:11 & 295 & 23002 & 19.37 & 0.13 \\
\hline 00214805000 & UVOT/B & 2006-06-14 19:12:15 & 2006-06-14 19:17:15 & 295 & 23306 & 19.50 & 0.16 \\
\hline 00214805000 & UVOT/White & 2006-06-14 19:17:19 & 2006-06-14 19:22:19 & 295 & 23610 & 18.25 & 0.13 \\
\hline 00214805000 & UVOT/White & 2006-06-14 19:22:23 & 2006-06-14 19:27:23 & 295 & 23914 & 18.26 & 0.11 \\
\hline 00214805000 & UVOT/White & 2006-06-14 19:27:25 & 2006-06-14 19:28:46 & 80 & 24216.5 & 18.31 & 0.22 \\
\hline 00214805000 & UVOT/UVW1 & 2006-06-14 20:23:21 & 2006-06-14 20:38:21 & 886 & 27572 & 18.38 & 0.12 \\
\hline 00214805000 & UVOT/U & 2006-06-14 20:38:27 & 2006-06-14 20:43:27 & 295 & 28478 & 18.34 & 0.10 \\
\hline 00214805000 & UVOT/U & 2006-06-14 20:43:31 & 2006-06-14 20:45:11 & 99 & 28782 & 18.45 & 0.21 \\
\hline 00214805001 & UVOT/UVW1 & 2006-06-15 00:01:34 & 2006-06-15 02:54:47 & 396 & 40665.5 & 19.02 & 0.26 \\
\hline 00214805001 & UVOT/U & 2006-06-15 00:03:54 & 2006-06-15 02:55:59 & 197 & 40805.5 & 18.76 & 0.17 \\
\hline 00214805001 & $\mathrm{UVOT} / B$ & 2006-06-15 00:05:05 & 2006-06-15 02:57:10 & 197 & 40876.5 & 19.73 & 0.19 \\
\hline 00214805001 & UVOT/UVW2 & 2006-06-15 00:06:19 & 2006-06-15 03:01:45 & 794 & 40950 & 18.74 & 0.46 \\
\hline 00214805001 & UVOT/V & 2006-06-15 00:10:53 & 2006-06-15 03:02:57 & 197 & 41224 & 19.23 & 0.28 \\
\hline 00214805001 & UVOT/UVM2 & 2006-06-15 00:12:04 & 2006-06-15 03:06:01 & 527 & 41295.5 & 18.65 & 0.54 \\
\hline 00214805001 & UVOT/UVW1 & 2006-06-15 04:29:34 & 2006-06-15 07:44:28 & 375 & 56745 & 19.17 & 0.26 \\
\hline 00214805001 & UVOT/U & 2006-06-15 04:31:52 & 2006-06-15 07:45:29 & 187 & 56883.5 & 19.29 & 0.21 \\
\hline 00214805001 & $\mathrm{UVOT} / B$ & 2006-06-15 04:33:03 & 2006-06-15 07:46:31 & 187 & 56954 & 19.90 & 0.22 \\
\hline 00214805001 & UVOT/VW2 & 2006-06-15 04:34:16 & 2006-06-15 07:50:26 & 753 & 57027 & 19.63 & 0.27 \\
\hline 00214805001 & $\mathrm{UVOT} / V$ & 2006-06-15 04:38:49 & 2006-06-15 07:51:28 & 187 & 57300.5 & $>19.80$ & - \\
\hline 00214805001 & UVOT/UVM2 & 2006-06-15 04:40:02 & 2006-06-15 07:54:02 & 502 & 57373 & 18.75 & 0.33 \\
\hline 00214805001 & UVOT/UVW1 & 2006-06-15 09:18:34 & 2006-06-15 12:34:28 & 474 & 74085 & 19.35 & 0.22 \\
\hline 00214805001 & UVOT/U & 2006-06-15 09:20:52 & 2006-06-15 12:35:59 & 237 & 74223.5 & 19.33 & 0.19 \\
\hline 00214805001 & UVOT/B & 2006-06-15 09:22:04 & 2006-06-15 12:37:32 & 237 & 74295 & 20.35 & 0.27 \\
\hline 00214805001 & UVOT/UVW2 & 2006-06-15 09:23:15 & 2006-06-15 12:43:26 & 950 & 74366.5 & 19.75 & 0.60 \\
\hline 00214805001 & $\mathrm{UVOT} / V$ & 2006-06-15 09:27:49 & 2006-06-15 12:44:59 & 237 & 74640 & 19.77 & 0.35 \\
\hline 00214805001 & UVOT/UVM2 & 2006-06-15 09:28:60 & 2006-06-15 12:49:02 & 648 & 74711 & 19.04 & 0.37 \\
\hline 00214805001 & UVOT/UVW1 & 2006-06-15 14:07:33 & 2006-06-15 17:24:57 & 769 & 91424 & 19.68 & 0.23 \\
\hline 00214805001 & UVOT/U & 2006-06-15 14:11:51 & 2006-06-15 17:27:15 & 384 & 91682.5 & 19.61 & 0.18 \\
\hline 00214805001 & UVOT/B & 2006-06-15 14:14:04 & 2006-06-15 17:29:32 & 384 & 91815 & 20.48 & 0.23 \\
\hline 00214805001 & UVOT/UVW2 & 2006-06-15 14:16:18 & 2006-06-15 17:38:25 & 1540 & 91949.5 & 20.52 & 0.43 \\
\hline 00214805001 & $\mathrm{UVOT} / V$ & 2006-06-15 14:24:52 & 2006-06-15 17:40:44 & 384 & 92463 & $>20.30$ & - \\
\hline 00214805001 & UVOT/UVM2 & 2006-06-15 14:27:03 & 2006-06-15 17:47:03 & 1086 & 92594.5 & 19.78 & 0.60 \\
\hline 00214805001 & UVOT/UVW1 & 2006-06-15 18:56:33 & 2006-06-15 22:13:57 & 779 & 108764 & 20.45 & 0.32 \\
\hline 00214805001 & UVOT/U & 2006-06-15 19:01:03 & 2006-06-15 22:16:13 & 389 & 109034 & 20.72 & 0.35 \\
\hline 00214805001 & $\mathrm{UVOT} / B$ & 2006-06-15 19:03:19 & 2006-06-15 22:18:31 & 387 & 109170 & 20.77 & 0.27 \\
\hline 00214805001 & UVOT/UVW2 & 2006-06-15 19:05:37 & 2006-06-15 22:27:27 & 1560 & 109308 & 20.50 & 0.40 \\
\hline 00214805001 & UVOT/V & 2006-06-15 19:14:33 & 2006-06-15 22:29:45 & 389 & 109844 & $>20.30$ & - \\
\hline 00214805001 & UVOT/UVM2 & 2006-06-15 19:16:49 & 2006-06-15 22:36:01 & 1101 & 109980 & 20.47 & 0.74 \\
\hline
\end{tabular}


Table 2. continued.

\begin{tabular}{|c|c|c|c|c|c|c|c|}
\hline Sequence & Obs/Filter & Start time $(\mathrm{UT})$ & End time (UT) & Exposure (s) & Start time $^{a}$ & $\mathrm{mag}^{b}$ & err \\
\hline 00214805002 & UVOT/UVW2 & 2006-06-16 01:32:19 & 2006-06-17 21:03:49 & 9481 & 132510 & 21.25 & 0.19 \\
\hline 00214805002 & UVOT/UVW1 & 2006-06-16 00:08:07 & 2006-06-17 22:28:05 & 7050 & 127458 & 21.24 & 0.16 \\
\hline 00214805002 & UVOT/U & 2006-06-16 00:10:31 & 2006-06-17 22:30:21 & 3516 & 127602 & 21.66 & 0.26 \\
\hline 00214805002 & UVOT/ $B$ & 2006-06-16 00:11:44 & 2006-06-17 22:32:38 & 3520 & 127675 & $>22.50$ & - \\
\hline 00214805002 & UVOT/V & 2006-06-16 00:17:41 & 2006-06-17 22:43:51 & 3520 & 128032 & 21.42 & 0.34 \\
\hline 00214805002 & UVOT/UVM2 & 2006-06-16 00:18:54 & 2006-06-17 22:50:02 & 9880 & 128105 & 21.51 & 0.21 \\
\hline 00214805003 & UVOT/UVW2 & 2006-06-18 00:13:06 & 2006-06-20 21:20:60 & 13506 & 300557 & 22.74 & 0.31 \\
\hline 00214805003 & UVOT/UVW1 & 2006-06-18 00:03:41 & $2006-06-2022: 44: 55$ & 10151 & 299992 & 22.71 & 0.36 \\
\hline 00214805003 & UVOT/U & 2006-06-18 00:08:19 & 2006-06-20 22:47:13 & 4937 & 300270 & 22.20 & 0.34 \\
\hline 00214805003 & UVOT/B & 2006-06-18 00:10:42 & 2006-06-20 22:49:30 & 4741 & 300413 & $>22.70$ & - \\
\hline 00214805003 & UVOT/V & 2006-06-18 00:22:20 & 2006-06-20 23:00:48 & 4674 & 301111 & $>21.70$ & - \\
\hline 00214805003 & UVOT/UVM2 & 2006-06-18 00:24:42 & 2006-06-20 23:07:02 & 12910 & 301253 & 22.73 & 0.33 \\
\hline 00214805007-09 & UVOT/V & $2006-06-24 \quad 15: 27: 35$ & 2006-06-26 23:18:25 & 11028 & 873826 & $>22.10$ & - \\
\hline 00214805008-09 & UVOT $/ B$ & $2006-06-2500: 50: 17$ & $2006-06-26$ 23:30:02 & 6959 & 907588.5 & $>23.10$ & _ \\
\hline 00214805011-27 & UVOT/White & 2006-06-27 12:21:24 & 2006-07-10 23:26:12 & 9012 & 1121855 & 22.38 & 0.22 \\
\hline 00214805008-35 & UVOT/U & 2006-06-25 00:44:08 & 2006-08-02 00:00:02 & 184470 & 907219 & 23.70 & 0.23 \\
\hline 00214805008-37 & UVOT/UVW1 & $2006-06-2500: 56: 30$ & 2006-08-07 16:49:01 & 17548 & 907961.5 & $>23.10$ & - \\
\hline 00214805036-37 & UVOT/UVM 2 & 2006-08-03 00:08:56 & 2006-08-07 $16: 47: 48$ & 5773 & 4274707 & $>22.50$ & - \\
\hline 00214805036-37 & UVOT/UVW2 & 2006-08-03 01:58:13 & 2006-08-07 21:38:01 & 3802 & 4281264 & $>22.50$ & - \\
\hline
\end{tabular}

\footnotetext{
${ }^{a}$ Seconds since the trigger. ${ }^{b}$ Magnitude corrected for Galactic extinction.
} 